\title{
Pseudospectral Renormalization Method for Solitons in Quasicrystal Lattice with the Cubic-Quintic Nonlinearity
}

\author{
Nalan Antar \\ Department of Mathematics, Istanbul Technical University, Maslak, 34469 Istanbul, Turkey \\ Correspondence should be addressed to Nalan Antar; antarn@itu.edu.tr
}

Received 31 March 2014; Revised 21 July 2014; Accepted 22 July 2014; Published 10 September 2014

Academic Editor: Keshlan S. Govinder

Copyright ( 2014 Nalan Antar. This is an open access article distributed under the Creative Commons Attribution License, which permits unrestricted use, distribution, and reproduction in any medium, provided the original work is properly cited.

\begin{abstract}
We modified the spectral renormalization method as the pseudospectral renormalization method in order to find the localized solutions. The pseudospectral renormalization method can be applied to a large class of problems including different homogeneities. Using this computational method, we demonstrate the existence of two different solitons in optical media described by the selffocusing cubic and the self-defocusing quintic nonlinear Schrödinger equation with quasicrystal lattice. It is shown that there are two different lattice solitons corresponding to the first and the second renormalization factors for the self-focusing cubic and the self-defocusing quintic model. However, the self-focusing quintic nonlinearity without optical lattice does not support two different solitons. We showed that the lattice solitons corresponding to the first and the second renormalization factors have the same powers and amplitudes. We also demonstrate that quintic nonlinearity supports bistable solitons by adding the optical lattice such as a quasicrystal lattice. The linear and nonlinear stabilities of these solitons are investigated using direct simulation of the nonlinear Schrödinger equation with the cubic-quintic nonlinearity and its linearized equation.
\end{abstract}

\section{Introduction}

Optical solitons, that is, localized waves, maintain their profile in nonlocal optical media due to the balance between the group-velocity dispersion, diffraction, and nonlinear selfmodulation. Therefore, there have been a great deal of theoretical and experimental investigations in models based on nonlinear Schrödinger equation (NLS) or Gross-Pitaevskii equation with periodic potential, cubic, and quintic nonlinearities [1-8]. Solitons in media based on the cubic nonlinear Schrödinger equation are unstable in two and three dimensions. In fact the collapse also occurs in one-dimensional (1D) NLS equation with self-focusing quintic term [9]. Different strategies have been used to achieve stabilization of the solitons including optical lattices and competing nonlinearities such as ones represented by combinations of cubic-quintic (CQ) and quadratic-cubic types.

In recent years there has been considerable interest in studying solitons in system with periodic potentials or lattices, in particular, those that can be generated in nonlinear optical materials $[10,11]$ in order to get stable solitons. However, the external potential of complex systems can be much more general and physically richer than a periodic lattice. For example, atomic crystals can have various irregularities, such as defects and edge dislocations, and also quasicrystal structures, which have long-range orientational order but no translational symmetry $[12,13]$.

The optical lattices, such as periodic and quasicrystal lattices, are not necessary for stability of the solitons in the self-focusing cubic media [14]. The model with cubic and quintic nonlinearities and with quasicrystal lattice can be a crucial factor for stabilization of the solitons. Competing selffocusing cubic and self-defocusing nonlinearities stabilize 2D solitons with and without external potential but in the case of self-focusing cubic and self-focusing quintic nonlinearities solitons collapse without optical lattice. The optical lattice with self-focusing cubic and self-focusing quintic nonlinearities stabilizes the solitons.

The nonlinear Schrödinger equation with a certain class of nonlinearities can also support multistable soliton solutions. This means that more than one amplitude and speed of propagation of the soliton may exist and the powers of the solitons can be the same or different. For example, for kerr nonlinearity, nonlinear Schrödinger equation does 
not support the bistable solitons. The nonlinear Schrödinger equation with the cubic and quintic nonlinearity is universal mathematical model describing many physical situations. It arises in plasma physics $[15,16]$ and condensed matter physics [17] and Bose-Einstein condensation [18, 19]. Cubic and quintic nonlinearities support the bistable solitons. Without the optical lattices, the quintic nonlinearity does not support bistable solitons but with the optical lattices such as quasicrystals supports bistable solitons.

The solitons as discussed above arise in a situation where the pulse does not exchange energy with the fiber. These solitons are called conservative solitons. Dissipative solitons can occur in a variety of physical, chemical, and biological systems [20]. In contrast to these familiar conservative solitons, gain and loss play an essential role in the formation of dissipative solitons. Recently, dissipative localized states with shield-like phase structure were examined by Clerc et al. in [21]. Pseudospectral method can also be used to compute the dissipative localized nonlinear modes.

The aim of this work is to study the effect of competition between cubic and quintic nonlinearities on the existence of bistable solitons with the optical lattice such as quasicrystal lattices. Linear and nonlinear stability properties of lattice solitons centered at the maximum of the quasicrystal lattice are investigated. The paper is organized as follows. First we introduce the pseudospectral renormalization method and show the existence of lattice solitons at the maximum of the quasicrystal lattice for both the cubic-quintic and the quintic models. The nonlinear and linear stability properties of these solitons are studied by direct computations of nonlinear Schrödinger equation with cubic-quintic nonlinearities where the initial conditions are taken to be the lattice solitons with $\% 1$ perturbation.

\section{Pseudospectral Renormalization Method}

We consider the propagation of light beams along the $z$ axis in a cubic-quintic medium with a transverse refractiveindex modulation. Dynamics of the beam can be described by the $(2+1)$ dimensional nonlinear Schrödinger with the cubic-quintic nonlinearity equation for the dimensionless field amplitude $q$ :

$$
i q_{z}+\Delta u+\alpha|q|^{2} q+\beta|q|^{4} q+V(x, y) q=0 .
$$

In optics, $q(x, y, z)$ corresponds to the complex-valued, slowly varying amplitude of the electric field in the $x y$ plane propagating in the $z$ direction; the parameters $\alpha$ and $\beta$ represent the cubic and the quintic nonlinear terms. In general, they are complex quantities but in this work, we do not consider the dissipative terms. We take these parameters as real. $V(x, y)$ is the external potential that can be written as the intensity of a sum of $N$ phase-modulated plane waves; that is, (see [22])

$$
V_{N}(x, y)=\frac{V_{0}}{N^{2}}\left|\sum_{n=0}^{N-1} e^{i\left(k_{x} x+k_{y} y\right)}\right|^{2},
$$

where $V_{0}$ is the peak depth of the potential; that is, $V_{0}=$ $\max _{x, y}[V(x, y)]$, and $\vec{k}_{n}$ is a wave vector defined by $\left(k_{x}, k_{y}\right)=$ $(K \cos (2 \pi n / N), K \sin (2 \pi n / N))$. The potentials for $N=$ $2,3,4,6$ yield periodic lattices that correspond to standard 2D crystal structures whereas $N=5,7$ correspond to quasicrystals. They have a local symmetry around the origin and long-range order but unlike periodic crystals are not invariant under spatial translations. In particular, the quasicrystal with $N=5$ is often called the Penrose tiling. Recently, Freedman et al. observed solitons in Penrose and other quasicrystal lattices generated by the optical induction method [23].

In this work, we will consider the lattice solitons centered at the maximum of a Penrose potential $(N=5)$ with the cubic-quintic nonlinearity. In Figure 1, contour image of the Penrose lattice for $V_{0}=3.5, K=1$ and diagonal cross section of the Penrose potential are displayed. As it is seen from Figure 1 there is only one global maximum at $x=0$.

We look for a soliton solution of (1) of the form

$$
q(x, y, z)=u(x, y) e^{i \mu z},
$$

where $u(x, y)$ is a real valued function and $\mu$ is the real propagation constant (eigenvalue). Substituting this form of solution into (1), the following nonlinear eigenequation is obtained:

$$
\Delta u+\left[-\mu+\alpha|u|^{2}+\beta|u|^{4}+V(x, y)\right] u=0 .
$$

This nonlinear eigenequation is supplemented with the boundary conditions

$$
u \longrightarrow 0, \quad|x|,|y| \longrightarrow \infty .
$$

Relaxation technique and the self-consistency method have been used to find the localized solitons arising in optics. Ablowitz and Musslimani [24] proposed a numerical scheme called spectral renormalization method for computing solitons in nonlinear wave guides. The essence of the method is to transform the governing equation into Fourier space and find a nonlinear nonlocal integral equation coupled with an algebraic equation. The coupling prevents the numerical scheme from diverging. The optical mode is then obtained from an iteration scheme, which converges rapidly. When we use the spectral renormalization method for different homogeneities such as saturable case, the convergence factor cannot be found explicitly. It has to use the root finding method such as Newton-Raphson method. We modified spectral renormalization method as pseudospectral renormalization method in order to find convergence factor explicitly from the governing equation. This method can efficiently be applied to a large class of problems including higher order nonlinear terms with different homogeneities such as saturable nonlinearity.

The spectral renormalization method can also be used to find the dissipative solitons. Ablowitz et al. used spectral renormalization method for solitons in dispersion-managed mode-locked lasers [25]. In order to get a solution whose amplitude does not grow indefinitely or tend to zero they introduced the renormalization factor which cannot be found explicitly. It has to use suitable root finding methods. With the use of pseudospectral renormalization method can be 


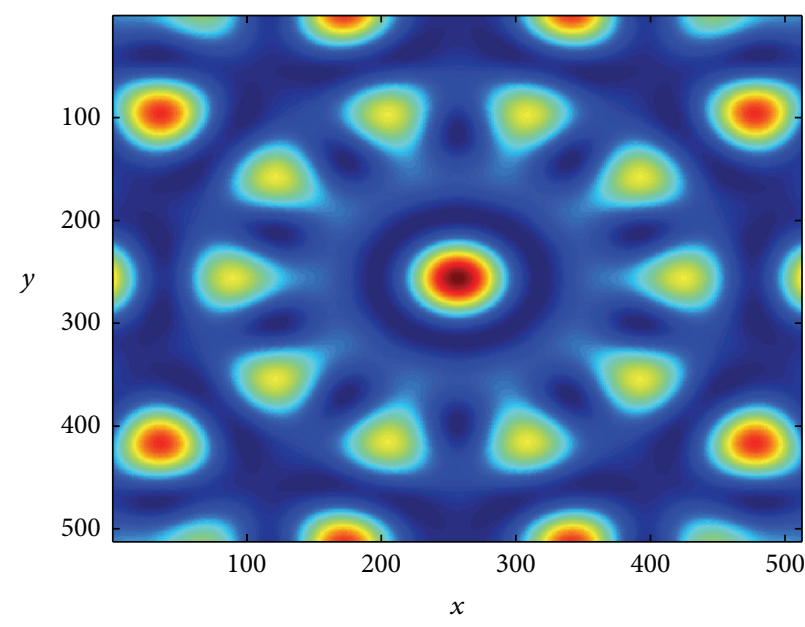

(a)

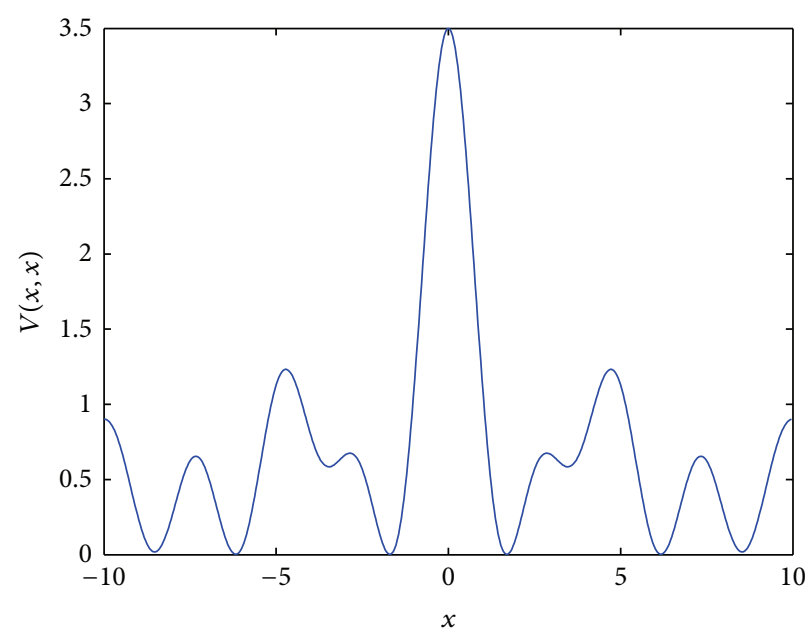

(b)

Figure 1: (a) Contour image of the Penrose potential with $V_{0}=3.5, K=1$. (b) Cross section of the Penrose potential along diagonal axis.

used for computing dissipative solitons without additional methods. But pseudospectral renormalization method only converges to the fundamental solitons. It diverges for excited states such as vortex solitons. Spectral renormalization method works well for the dipole and vortex solitons (see [26]).

We use a fixed-point pseudospectral computational method (pseudospectral renormalization method) to solve (4) as explained below. After applying the Fourier and inverse Fourier transformation to the Laplacian, $\Delta u=u_{x x}+u_{y y}$, we get the following equation:

$$
\begin{aligned}
& -\mathscr{F}^{-1}\left[\left(\mu+|k|^{2}\right) \mathscr{F}[u]\right] \\
& \quad+\alpha|u|^{2} u+\beta|u|^{4} u+V(x, y) u=0 .
\end{aligned}
$$

Here $\mathscr{F}$ and $\mathscr{F}^{-1}$ denote the Fourier and the inverse Fourier transformations, respectively. We introduce a new field variable $u(x, y)=\lambda w(x, y)$ in order to prevent the amplitude under iteration either growing without bound or tending to zero. Here $\lambda \neq 0$ is a constant to be determined. Then the function $w(x, y)$ satisfy the following equation:

$$
\begin{aligned}
& -\mathscr{F}^{-1}\left[\left(\mu+|k|^{2}\right) \mathscr{F}[w]\right] \\
& \quad+\alpha|\lambda|^{2}|w|^{2} w+\beta|\lambda|^{4}|w|^{4} w+V(x, y) w=0 .
\end{aligned}
$$

In order to find $|\lambda|$ which we call the renormalization factor, we multiply (7) by $w(x, y)$ and integrate over the entire space $x, y$; we obtain an equation for the renormalization factor as

$$
A|\lambda|^{4}+B|\lambda|^{2}+C=0
$$

where $A, B$, and $C$ are defined by

$$
\begin{array}{r}
A=\beta \int_{-\infty}^{\infty} \int_{-\infty}^{\infty} w\left[w|w|^{4}\right] d x d y, \\
B=\alpha \int_{-\infty}^{\infty} \int_{-\infty}^{\infty} w\left[w|w|^{2}\right] d x d y, \\
C=-\int_{-\infty}^{\infty} \int_{-\infty}^{\infty} w\left[F^{-1}\left[\left(\mu+|k|^{2}\right) F[w]\right]\right. \\
-V(x, y) w] d x d y .
\end{array}
$$

There are two roots of the equation given in (8) as

$$
\begin{aligned}
& |\lambda|_{1}^{2}=\frac{\left(-B+\sqrt{B^{2}-4 A C}\right)}{2 A}, \\
& |\lambda|_{2}^{2}=\frac{\left(-B-\sqrt{B^{2}-4 A C}\right)}{2 A} .
\end{aligned}
$$

In this work, we will call $|\lambda|_{1}^{2}$ as the first renormalization factor and $|\lambda|_{2}^{2}$ as the second renormalization factor. The solution to (6) is obtained by iterating as follows:

$$
\begin{aligned}
& w_{n+1} \\
& =\mathscr{F}^{-1}\left\{\frac{\left(\mathscr{F}\left[\alpha\left|\lambda_{n}\right|_{1,2}^{2}\left|w_{n}\right|^{2} w_{n}+\beta\left|\lambda_{n}\right|_{1,2}^{2}\left|w_{n}\right|^{4} w_{n}+V(x, y) w_{n}\right]\right)}{\left(\mu+|k|^{2}\right)}\right\}
\end{aligned}
$$

which is subject to the additional constraint where $\operatorname{Im}\left(\lambda_{n}\right)=$ 0 .

It has been found that this method often prevents the numerical scheme from diverging. Thus, the soliton is obtained from a convergent iterative scheme. The initial starting point $w_{0}(x, y)$ is typically chosen to be a Gaussian centered around one of the lattice's critical points. The iteration 


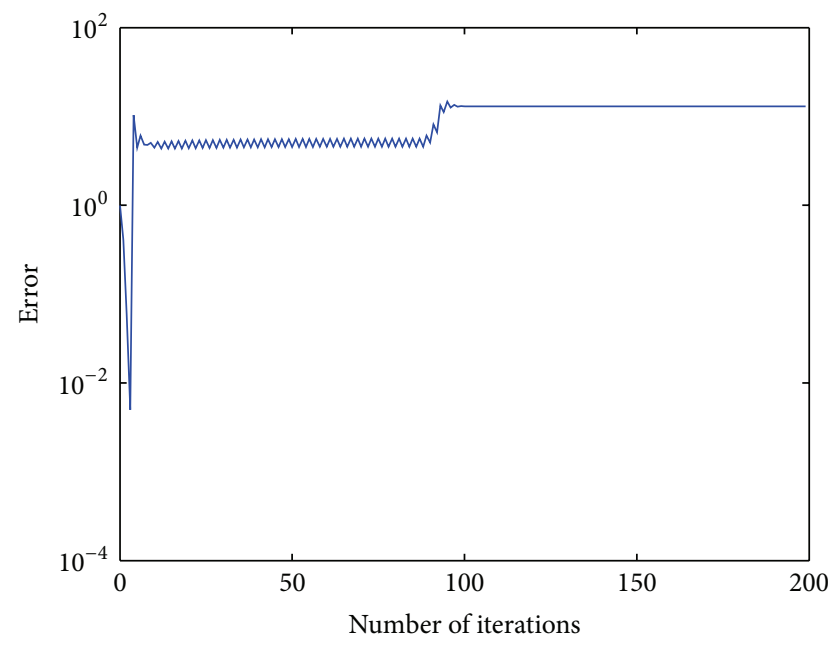

(a)

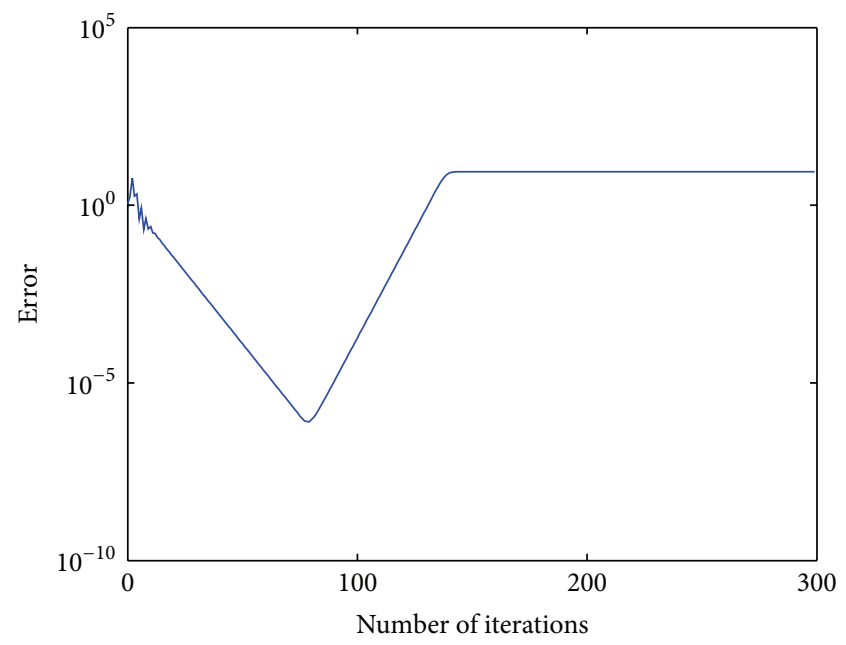

(b)

FIGURE 2: Convergence analysis for lattice soliton corresponding to the first renormalization factor with $\mu=1.29$; (b) convergence analysis for lattice soliton corresponding to the second renormalization factor with $\mu=1.49$.

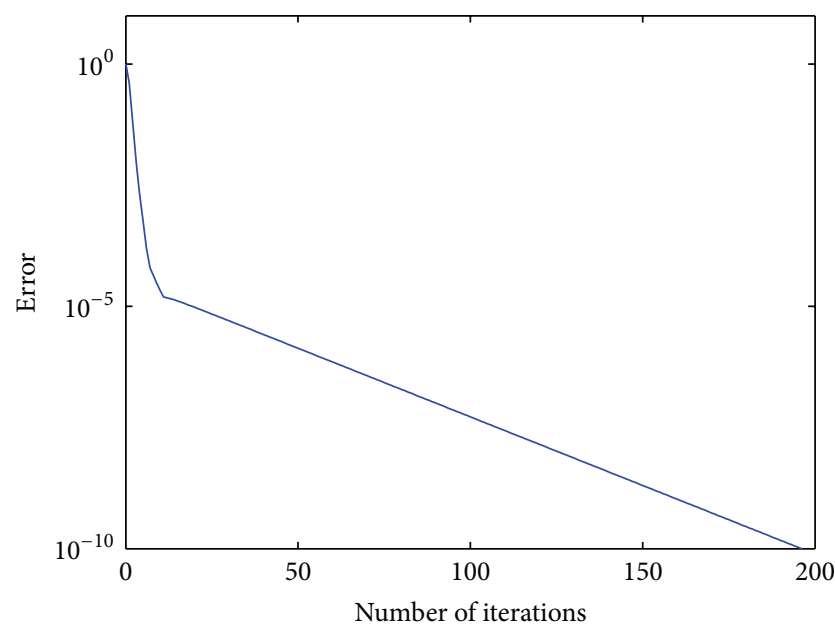

(a)

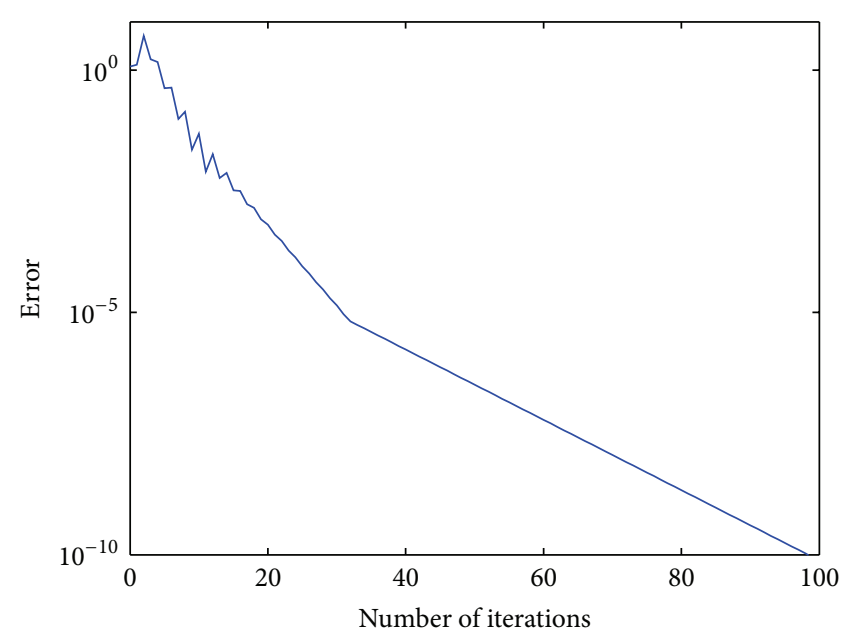

(b)

FIGURE 3: Convergence analysis for lattice soliton corresponding to the first renormalization factor with $\mu=1.3$; (b) convergence analysis for lattice soliton corresponding to the second renormalization factor with $\mu=1.5$.

continues until the relative error $\lambda_{\text {error }}=\left|\lambda_{n+1} / \lambda_{n}-1\right|$, $w_{\text {error }}=\left|w_{n+1}-w_{n}\right|$ reaches $10^{-10}$. We also check whether (7) satisfies or not for each spectral renormalization factors. We define error in semilog scale and with Euclidian norm as follows:

$$
\begin{aligned}
\text { error }= & \|-\mathscr{F}^{-1}\left[\left(\mu+|k|^{2}\right) \mathscr{F}[w]\right] \\
& +\alpha|\lambda|^{2}|w|^{2} w+\beta|\lambda|^{4}|w|^{4} w+V(x, y) w=0 \| .
\end{aligned}
$$

Convergence is usually obtained quickly.

\section{Lattice Solitons in the Cubic-Quintic Model}

In this section, we show the existence of the lattice solitons centered at the lattice maximum of the Penrose potential. We first investigate the self-focusing cubic and the self-defocusing quintic model. Therefore we set the cubic and quintic nonlinear terms as $\alpha=1$ and $\beta=-0.2$. The numerical calculations show that the lattice solitons obtained by the use of the first renormalization factor and the second renormalization factor exist in the band gap given by

$$
1.3 \leq \mu \leq 1.7, \quad 1.5 \leq \mu \leq 1.7 .
$$

Convergence is obtained quickly when the lattice solitons are strongly localized in these band gaps. Beyond the band gap, the lattice solitons can be found but we could not achieve the convergence or the lattice solitons become the imaginary. Numerical evidence with regard to the interval values in (13) is shown in Figures 2 and 3 for both the first and the second renormalization factors.

We showed that when the propagation constants are smaller than 1.3 for the first renormalization factor and 1.5 for the second renormalization factor the convergence cannot be obtained (see Figures 2(a) and 2(b)) but convergence is 


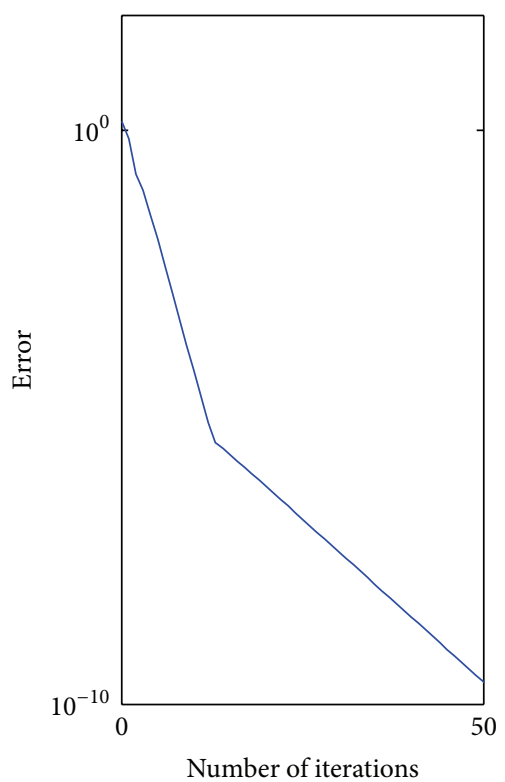

(a)

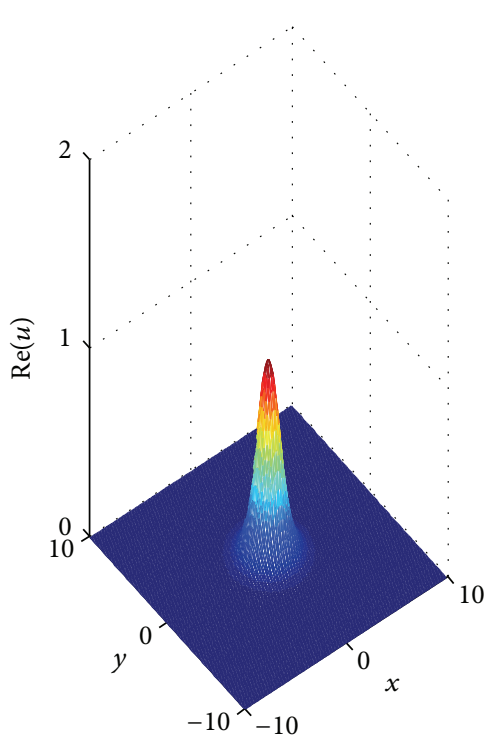

(b)

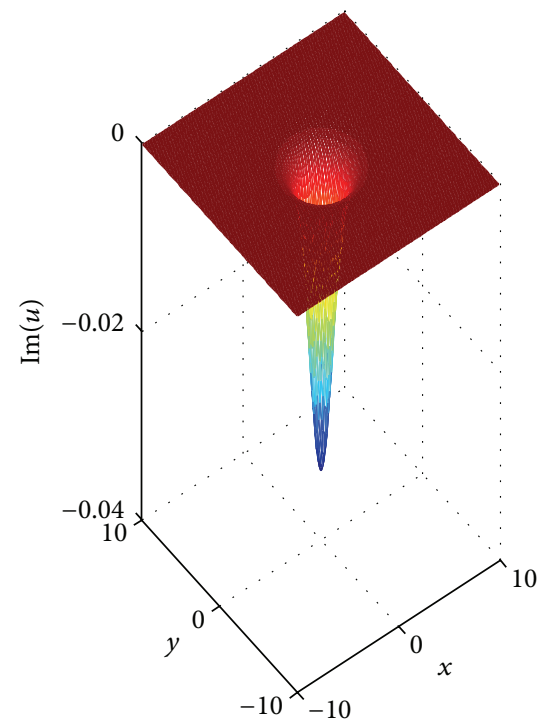

(c)

FIGURE 4: Convergence analysis for lattice soliton corresponding to the first renormalization factor with $\mu=1.71$.

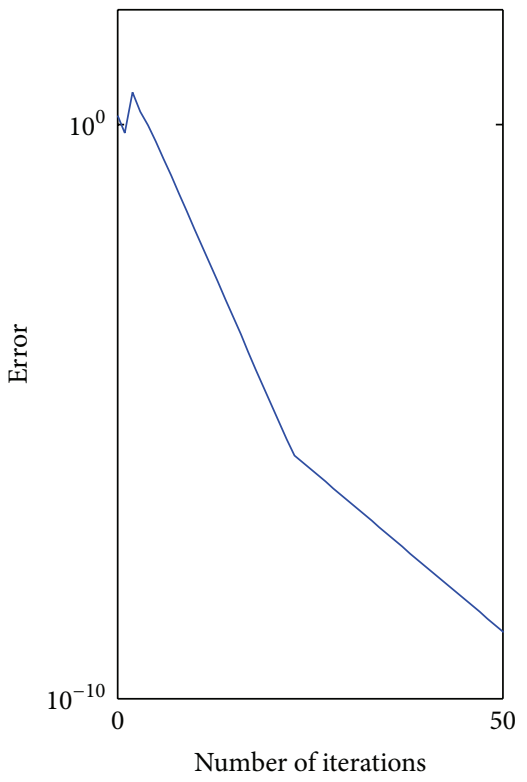

(a)

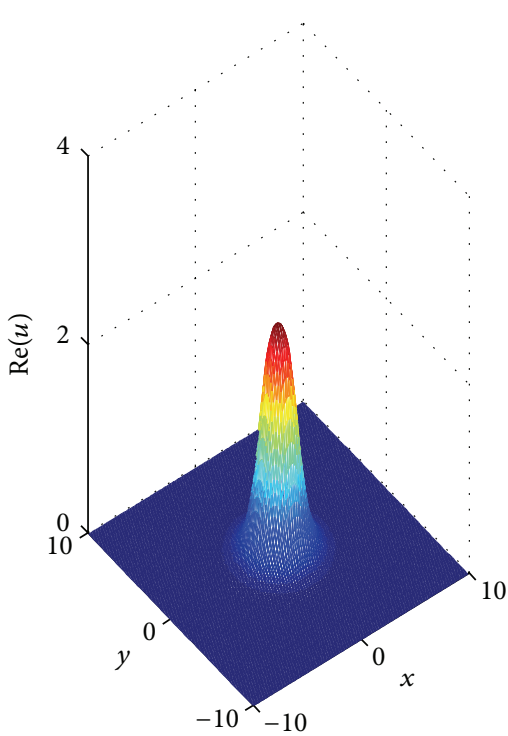

(b)

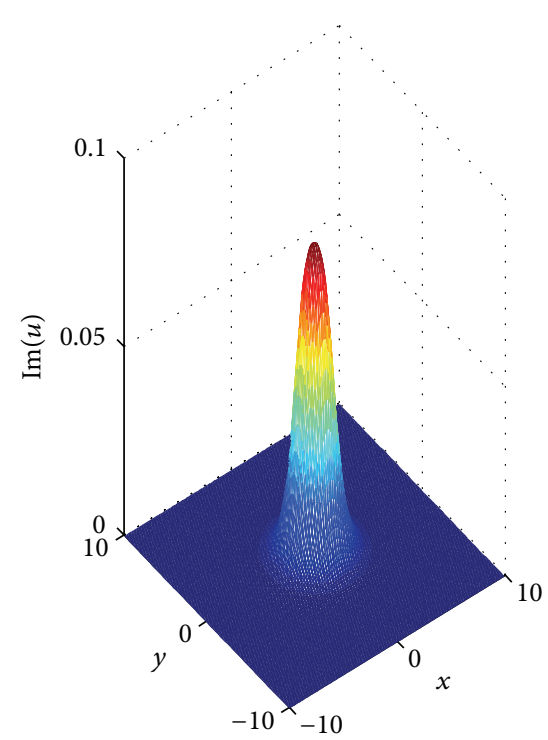

(c)

FIGURE 5: Convergence analysis for lattice soliton corresponding to the second renormalization factor with $\mu=1.71$.

obtained very quickly at the propagation constants $\mu=1.3$ and $\mu=1.5$ for both the first and the second renormalization factors (see Figures 3(a) and 3(b)).

We numerically found the lattice solitons corresponding to the first and the second renormalization factors with $\mu=$ 1.71. These solitons should be real but, in Figures 4 and 5, we demonstrated that when the propagation constant is bigger than 1.7 for both the first and the second renormalization factors the convergence is obtained very quickly but the lattice solitons become imaginary.
The lattice solitons obtained by the use of the first renormalization factor have a larger gap size compared with the lattice solitons obtained by the second renormalization factor.

Non-Kerr nonlinearities have new effects in soliton dynamics and suggest new possibilities for optical lattice applications. The cubic-quintic nonlinearity gives bistability and multistability of soliton families. In order to see this, we define the total power of the soliton given by

$$
P=\int_{-\infty}^{\infty} \int_{-\infty}^{\infty}|u(x, y)|^{2} d x d y .
$$




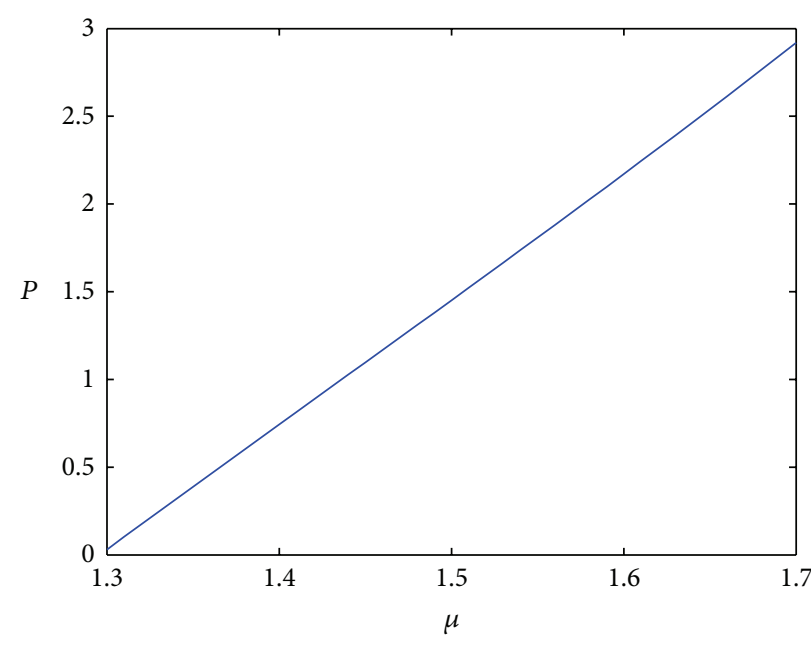

(a)

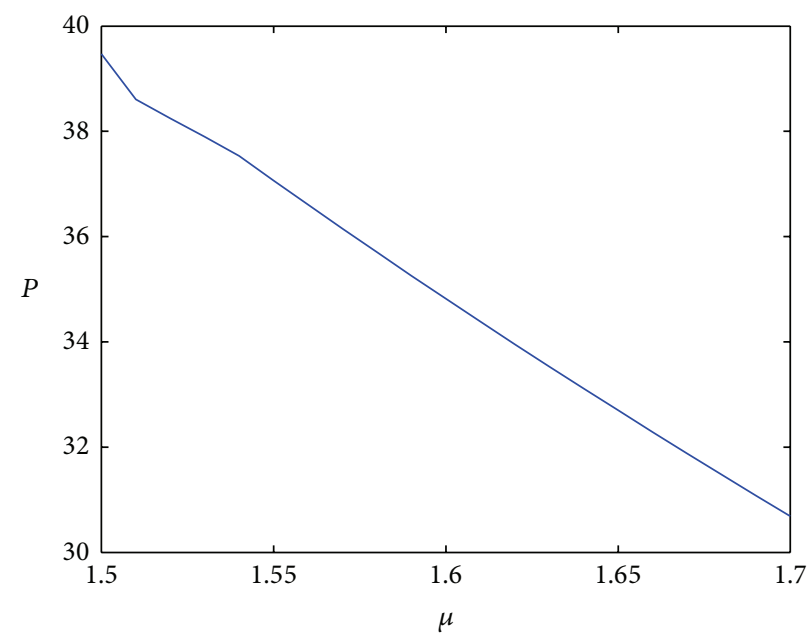

(b)

Figure 6: The power of the lattice solitons for the self-focusing cubic and the self-focusing quintic model versus the propagation constant for the Penrose potential. (a) The power of the solitons obtained by the use of the first renormalization factor versus $\mu$. (b) The power of the solitons obtained by the use of the second renormalization factor versus $\mu$.

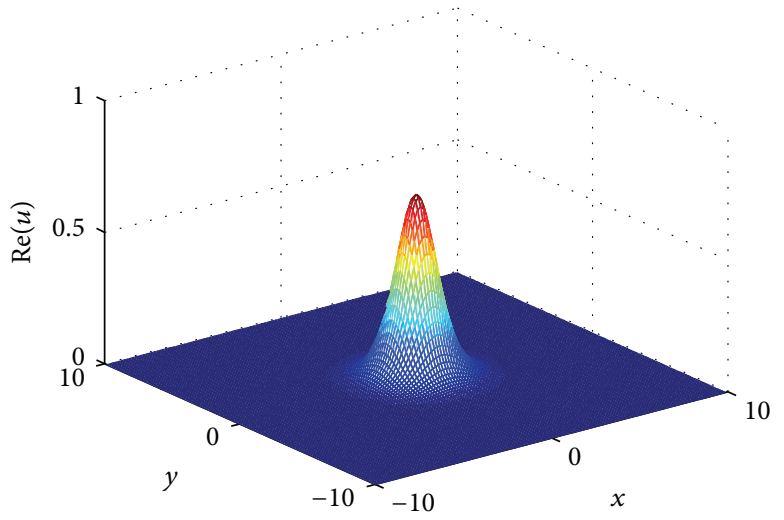

(a)

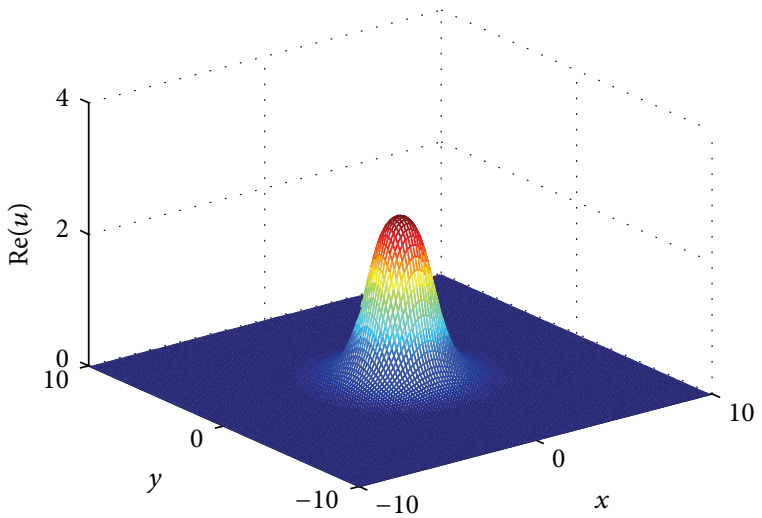

(b)

FIGURE 7: (a) Real part of the lattice soliton profile corresponding to the first renormalization factor with the Penrose potential, $V_{0}=3.5$, $K=1$. (b) Real part of the lattice soliton profile corresponding to the second renormalization factors with the Penrose potential, $V_{0}=3.5$, $K=1$.

We plotted the power of the lattice solitons obtained by the use of the first and second renormalization factors versus the propagation constant in the range of the band gap.

As it is seen from Figure 6, two types of the solitons are found by the use of two spectral renormalization factors for the Penrose potential. In a certain interval of variation of $\mu$, two different solitons are found for a given value of the propagation constant, which correspond to different soliton's profiles and different value of total power $P$. The typical lattice soliton's profiles corresponding to the first and the second renormalization factors are shown in Figure 7 for the propagation constant $\mu=1.5$. We also plot the cross sections along $x$-axis of the lattice solitons centered at the lattice maximum of the Penrose potential (see Figure 8). The maximum amplitude of the lattice soliton corresponding to the first renormalization factor is much smaller than the maximum amplitude of the lattice soliton corresponding to the second renormalization factor.

Unless the depth of the potential is too shallow $\left(V_{0}\right.$ is too small), all solitons feature the bistability with two different solitons but when the depth of the potential is too shallow, the cubic-quintic nonlinearities with the Penrose potential fail to produce the bistability solitons.

In the second case, we consider the self-focusing cubic and the self-focusing quintic nonlinearities. That is, we set the nonlinear parameters as $\alpha=1$ and $\beta=0.2$. All soliton families exist in the semi-infinite band gap for the first and second renormalization factors. In this case, solitons corresponding to the first renormalization factor are real but solitons corresponding to the second renormalization factor are purely imaginary. Their band gap structures are the same. In order to see whether the self-focusing cubic and the self-focusing 


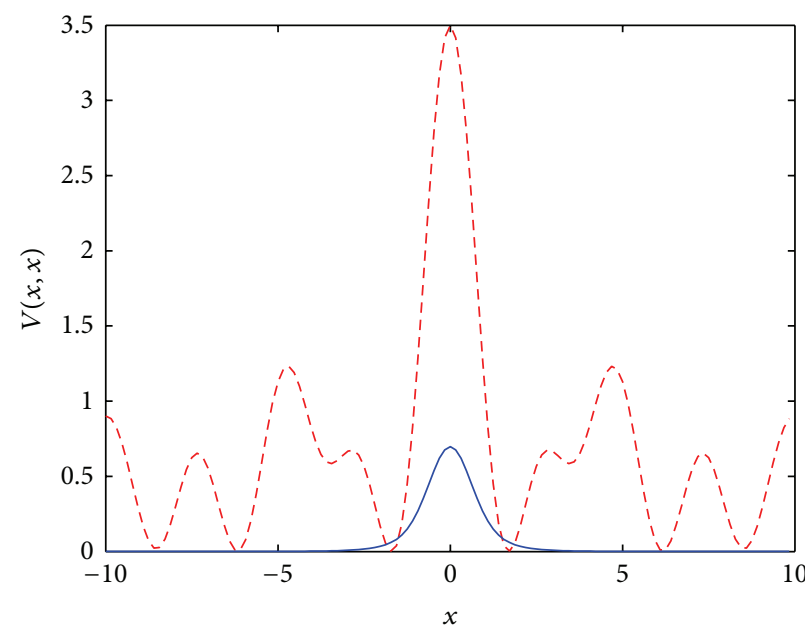

(a)

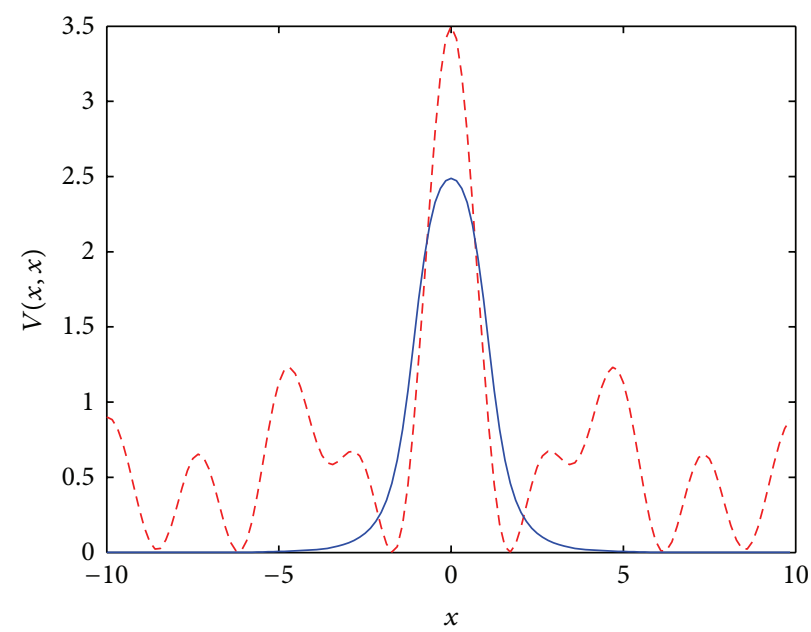

(b)

FIGURE 8: The lattice soliton profiles for the self-focusing cubic and the self-defocusing quintic model. (a) Cross section along $x$-axis of the lattice solitons corresponding to the first renormalization factor (solid line) superimposed on the underlying Penrose potential (dashed line). (b) Cross section along $x$-axis of the lattice solitons corresponding to the second renormalization factor (solid line) superimposed on the underlying Penrose potential (dashed line).

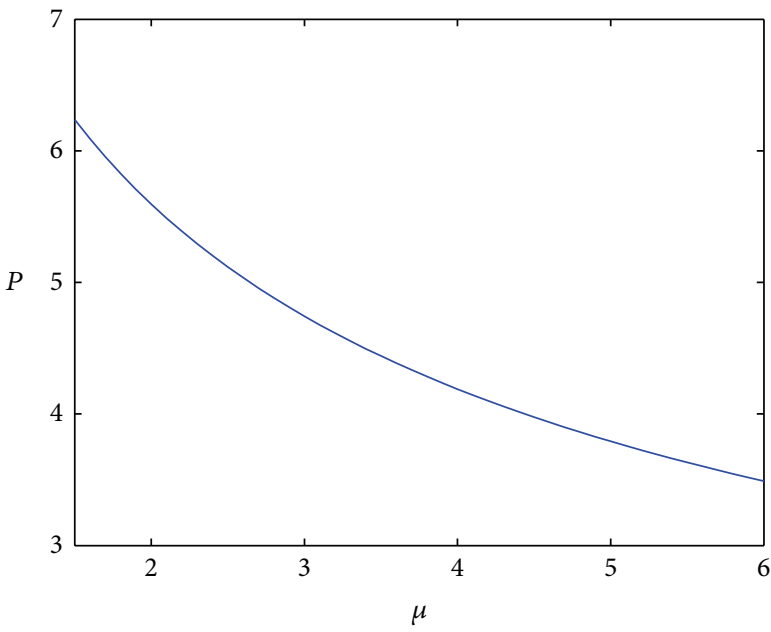

(a)

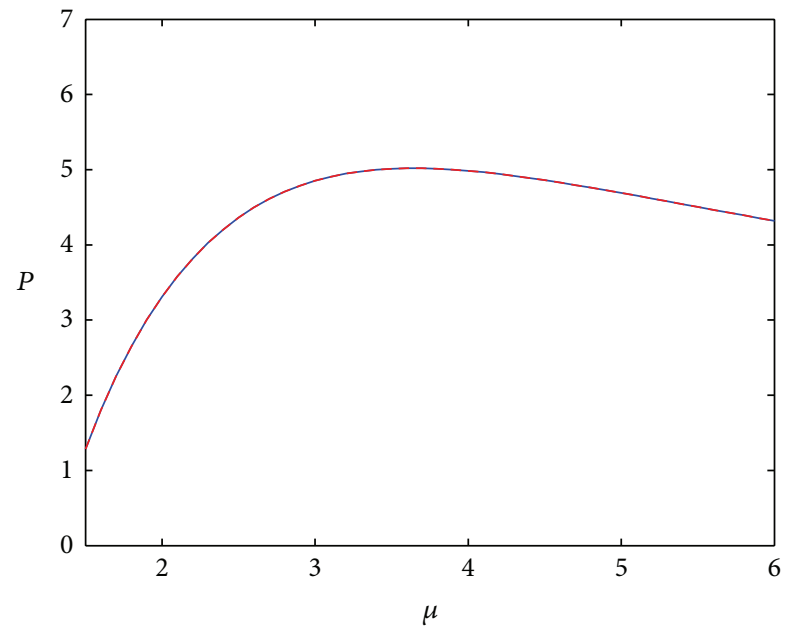

(b)

FIGURE 9: (a) The power of the solitons obtained by the use of the first and second renormalization factors versus $\mu$ without the optical lattice, $V_{0}=0$ for the self-focusing cubic and the self-focusing quintic model. (b) The power of the solitons obtained by the use of the first and second renormalization factors versus $\mu$ with the Penrose potential, $V_{0}=3.5$ for the self-focusing cubic and the self-focusing quintic model.

quintic terms produce two different solitons with the different wave number, we plotted the power versus the propagation constant (see Figure 9).

The self-focusing cubic and the self-focusing quintic model without the optical lattice do not support two different solitons since the power of the solitons decreases with the propagation constant (see Figure 9(a)). As it is seen from Figure 9 (b) the stability change point does exist in the model with the self-focusing cubic and the self-focusing quintic model combining the optical lattice such as the Penrose potential. The lattice soliton profiles for this model are displayed in Figure 10.
Typical examples of lattice solitons found in the selffocusing cubic and the self-focusing quintic model with the Penrose potential for $\mu=1.5$ and $\mu=5$ are represented in Figure 10. As it is seen from this figure, the lattice solitons get more steep after a point at which $d P / d \mu<0$.

\section{Lattice Solitons in the Quintic Model}

In this section, we consider the self-focusing quintic model and we set $\alpha=0$ and $\beta=1$ in (1). Lattice solitons corresponding to the first and second renormalization factors for the Penrose potential are found numerically with the Gaussian 


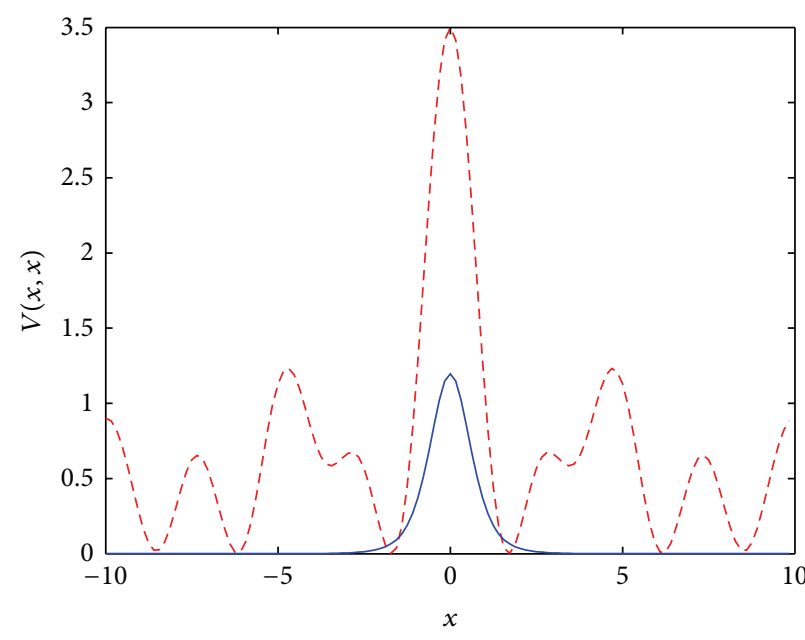

(a)

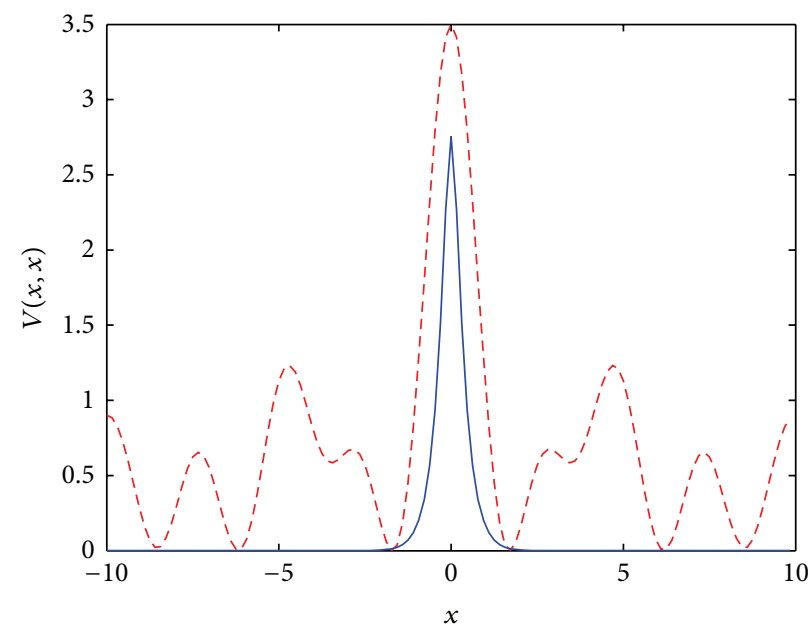

(b)

FIGURE 10: The lattice soliton profiles for the self-focusing cubic and the self-focusing quintic model. (a) Cross section along $x$-axis of the lattice solitons corresponding to the first renormalization factor (solid line) superimposed on the underlying Penrose potential (dashed line). (b) Cross section along $x$-axis of the lattice solitons corresponding to the first renormalization factor (solid line) superimposed on the underlying Penrose potential (dashed line).

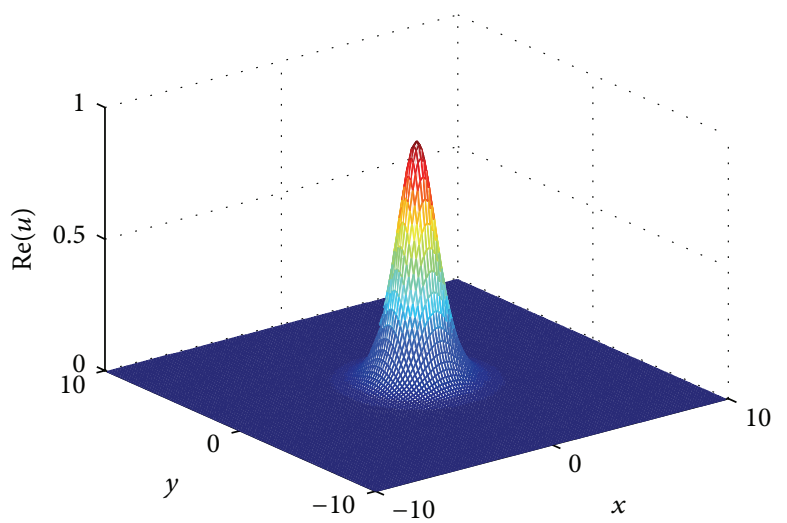

(a)

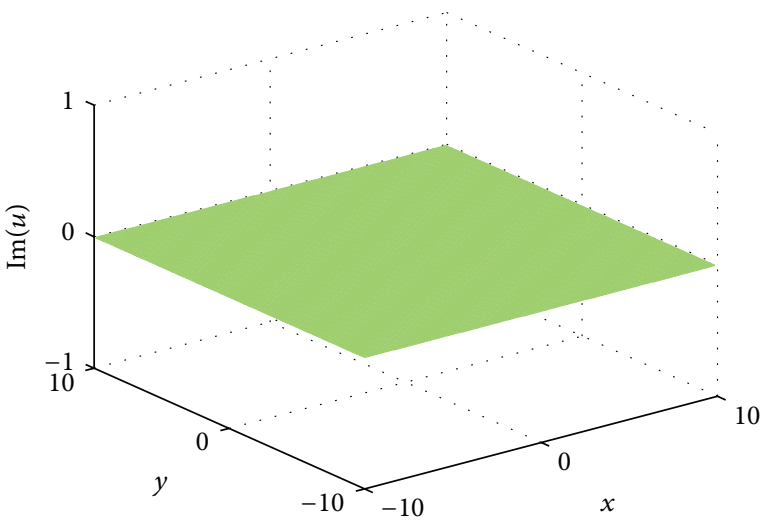

(b)

FIGURE 11: Lattice soliton profile corresponding to the first renormalization factor on the Penrose potential, all with $V_{0}=3.5, K=1$, and $\mu=$ 1.5 for the self-focusing quintic model. (a) Real part of the lattice soliton. (b) Imaginary part of the lattice soliton.

initial condition. The lattice solitons exist in the semi-infinite band gap. When the propagation constant increases the lattice solitons become singular. The profile of the lattice soliton corresponding to first renormalization factor can be seen in Figure 11. As you see from this figure, for the first renormalization factor the lattice soliton is purely real but for the lattice soliton corresponding to the second renormalization factor it is purely imaginary (see Figure 12) but these solitons have the same amplitudes and powers corresponding to the same propagation constant.

In order to see whether two different solitons exist in the quintic model with the Penrose potential, we plotted the power of the solitons versus the propagation constant with and without optical lattices. As it is seen from Figure 13(a), the power decreases with the propagation constant. This means that two different solitons cannot be observed in the self-focusing quintic model without the optical lattice.
However, for certain range of $P$ there are two different $\mu$ values to which correspond two differently proportioned lattice solitons centered at the maximum of the Penrose potential. Adding the Penrose potential and the self-focusing quintic nonlinearity supports two different solitons.

An example of a pair of lattice solitons found at a given different value of $\mu$ whose power is the same is displayed in Figure 14. Notice that when the propagation constant is bigger than 2.5 , the lattice solitons get narrower.

\section{Linear Stability of Lattice Solitons}

Until now, we have shown the existence of solitons at the lattice maximum of the Penrose potential. Now we address the critical question of linear stability of these solitons. For this, we linearized (1) around the lattice soliton obtained 


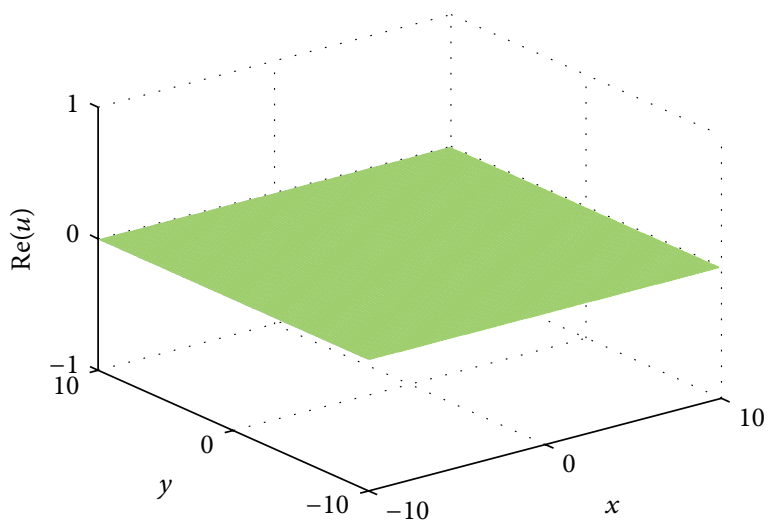

(a)

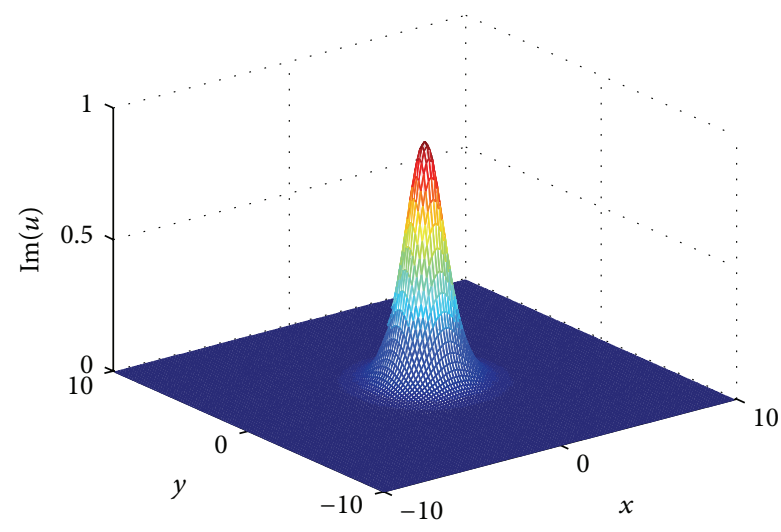

(b)

FIGURE 12: Lattice soliton profile corresponding to the second renormalization factor on the Penrose potential, with $V_{0}=3.5, K=1$, and $\mu=1.5$ for the self-focusing quintic model. (a) Real part of the lattice soliton. (b) Imaginary part of the lattice soliton.

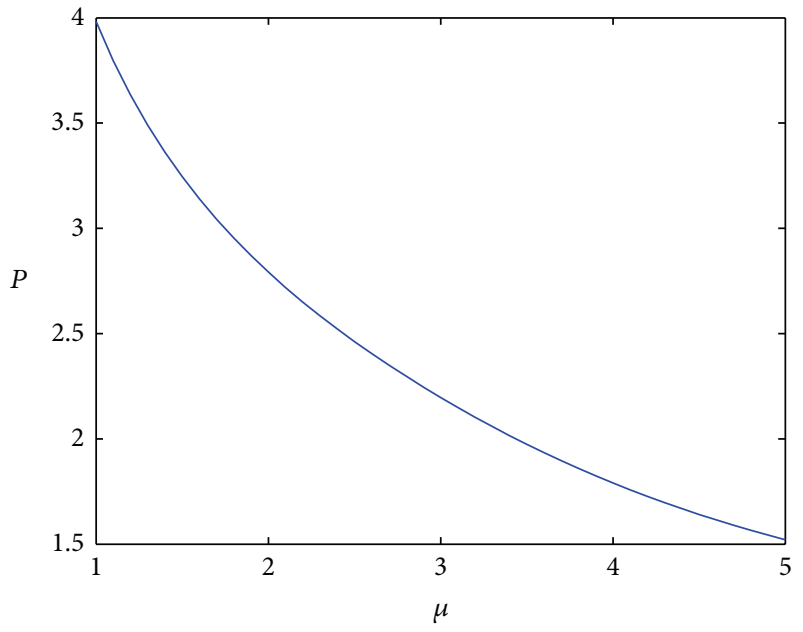

(a)

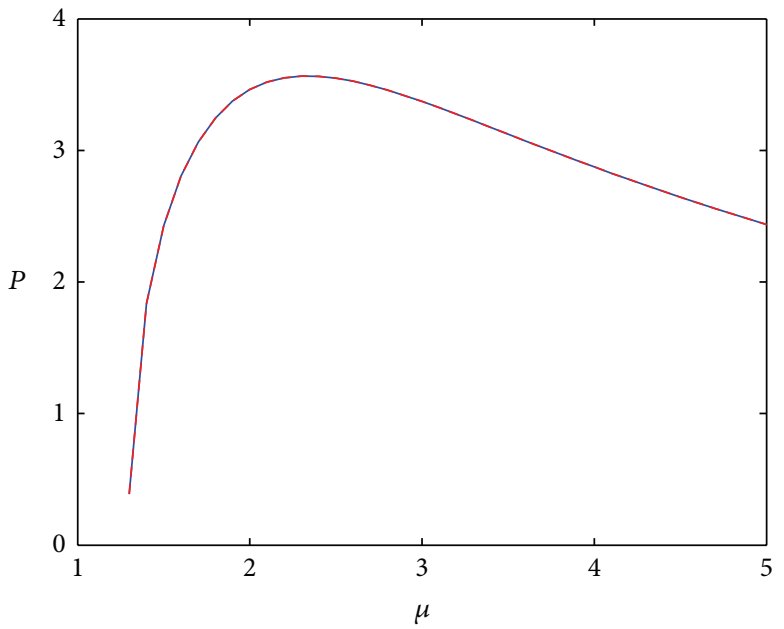

(b)

FIGURE 13: The total power of the lattice solitons versus propagation constant $\mu$ for the self-focusing quintic model. (a) Without external potential, $V_{0}=0$. (b) With the Penrose potential, $V_{0}=3.5$.

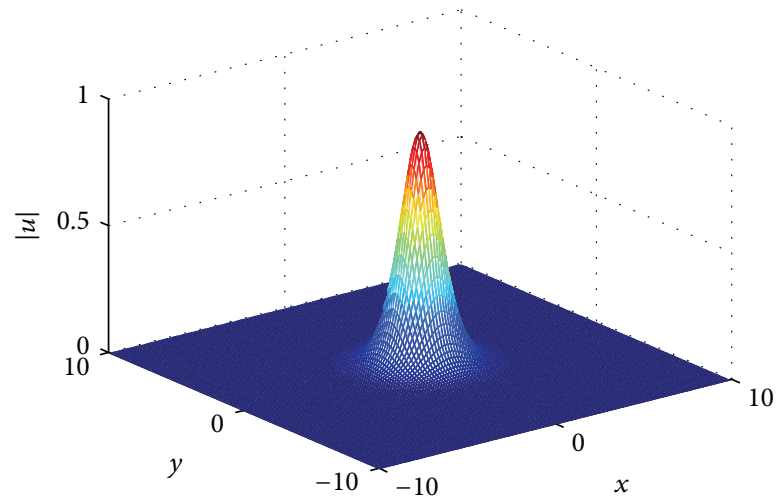

(a)

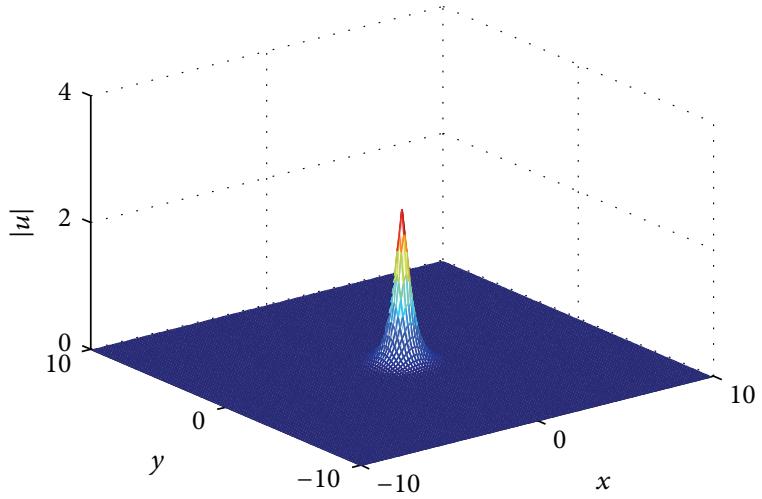

(b)

Figure 14: The lattice soliton profiles for the self-focusing quintic model: (a) for $\mu=1.5, P=2.4273$, (b) for $\mu=5, P=2.4273$. 
by the use of pseudospectral renormalization method. By denoting

$$
u=\exp (i \mu z)\left[u_{0}(x, y)+\epsilon \tilde{u}(x, y, z)\right],
$$

where $u_{0}(x, y)$ is the computed lattice soliton and $\epsilon \tilde{u}$ is the infinitesimal perturbation, where $\widetilde{\epsilon} \ll 1$, the linearized equation for $\tilde{u}$ is given by

$$
\begin{aligned}
i \frac{\partial \tilde{u}}{\partial z}+ & \frac{\partial^{2} \tilde{u}}{\partial x^{2}}+\frac{\partial^{2} \tilde{u}}{\partial y^{2}} \\
& +\left(-\mu+V(x, y)+2 \alpha\left|u_{0}\right|^{2}+3 \beta u_{0}^{2} u_{0}^{* 2}\right) \tilde{u} \\
& +\left(\alpha u_{0}^{2}+2 \beta u_{0}^{3} u_{0}^{*}\right) \widetilde{u}^{*}=0 .
\end{aligned}
$$

Starting from a white-noise initial condition, we simulated this linearized equation over a long distance with the Penrose potential. Finite differences on $\widetilde{u}_{x x}$ and $\widetilde{u}_{y y}$ and the fourth-order Runge-Kutta method to advance in $z$ are employed.

The power of the soliton plays an important role in determining the linear stability properties of the solitons. An important analytic result on soliton stability was obtained by Vakhitov-Kolokolov (VK) [27]. They proved, by use of the linearized perturbation equation, that a necessary condition for stability of the soliton $u(x ; \mu)$ is

$$
\frac{d P}{d \mu}>0
$$

that is, the soliton is stable only if its power increases with increasing propagation constant $\mu$. This condition is called the slope condition. In the next section, we will investigate the linear stability properties of the lattice solitons obtained in the previous section for the cubic-quintic and quintic models, respectively.

5.1. Linear Stability of Lattice Solitons in the Cubic-Quintic Model. In this section, in order to see whether VakhitovKolokolov criteria are valid for the cubic-quintic model, we plot the total power versus the propagation constant for lattice solitons corresponding to the first renormalization factor and the second renormalization factor (see Figure 6). As it is seen from Figure 6, the power of the solitons corresponding to the first renormalization factor increases with the propagation constant but the power of the solitons corresponding to the second renormalization factor decreases with the propagation constant. That is, the lattice solitons obtained by the use of the first renormalization factor should be linearly stable but the lattice solitons obtained by the use of the second renormalization factor should be linearly unstable. In order to see this, we perform direct computation of (16) where the initial condition is $1 \%$ random noise in amplitude and phase. We plotted the maximum amplitudes of these solitons versus the propagation distance $z$ (see Figure 15). As seen from Figure 15, the solitons corresponding to the first and second renormalization factors are linearly unstable since their maximum of the amplitudes increases with the propagation distance $z$.
The VK criteria produce accurate results for the lattice solitons corresponding to the second renormalization factor but the VK criteria did not produce accurate results for the lattice solitons corresponding to the first renormalization factor.

5.2. Linear Stability of Lattice Solitons in the Quintic Model. In this previous section, we found that there are two different solitons corresponding to the first and the second renormalization factors. They are linearly unstable. In this section, we investigate the linear stability properties of the solitons for quintic model. The solution of (1) in 1D case with $\alpha=0$ and without external potential $V_{0}=0$ can be given as [28]

$$
u(x)=\frac{(3 \mu /|\beta|)^{1 / 4}}{\sqrt{\cosh (2 \sqrt{\mu x})}} .
$$

The critical power of the soliton is $P_{\mathrm{cr}}=(\pi / 2) \sqrt{3 /|\beta|} \approx$ 2.7. The similar behavior is valid for two-dimensional problem. It is possible to predict the linear stability of the lattice solitons on the basis of the Vakhitov-Kolokolov (VK) criterion, a necessary condition for stability which is given by $d P / d \mu>0$. The Vakhitov-Kolokolov fails to produce accurate results for the quintic model. Even if the power versus the propagation constant increases, the lattice solitons are linearly unstable since their power exceeds the critical power. As it is seen from Figure 13(a) the power increases with the propagation constant without lattice and the power exceeds the critical power.

We investigate the linear properties of the lattice solitons with the Penrose potential and without potential. We plot the peak amplitude of the lattice solitons versus the propagation distance (see Figure 16).

Figure 16(a) shows that the peak amplitude of the lattice soliton without potential is nearly constant (typically $z<$ 0.2 ), followed by exponential growth. Unstable dynamics is also observed for the case of the lattice soliton obtained with the Penrose potential (see Figure 16(b)). None of the lattice solitons with and without Penrose potential is linearly stable.

\section{Nonlinear Evolution of Lattice Solitons under Weak Perturbations}

In this section, we examine the nonlinear evolution of lattice solitons under weak perturbation. To investigate the nonlinear evolution of the lattice solitons, we directly compute (1) over a long distance (finite difference method was used on $q_{x x}$ and $q_{y y}$ and fourth-order Runge-Kutta method to advance in $z$ ). The initial conditions were taken to be the lattice soliton with 0.01 perturbation in the amplitude and phase.

The soliton is unstable only if its power decreases with increasing propagation constant $\mu$. This condition is called the slope condition.

The lattice solitons can become unstable in two ways: focusing instability or drift instability [29].

(a) If the slope condition, that is, power decreases with increasing propagation constant $\mu$, is not satisfied, this leads to a focusing instability. 


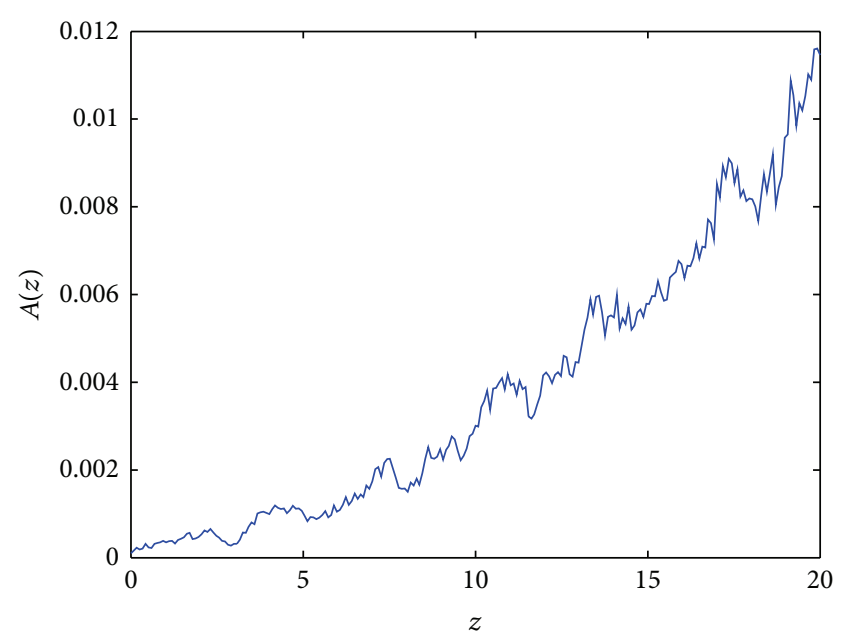

(a)

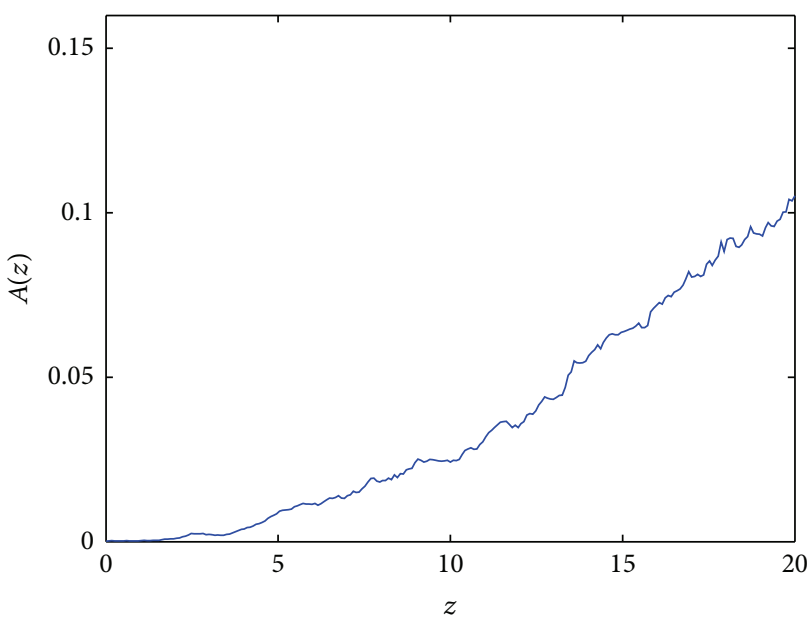

(b)

FIGURE 15: Linear evolution of the lattice soliton for the self-focusing cubic and the self-defocusing quintic model: peak amplitude $A(z)=$ $\max _{x, y}|\tilde{u}(x, y, z)|$ of the solitons as the function of $z$, (a) for the first renormalization factor and (b) for the second renormalization factor.

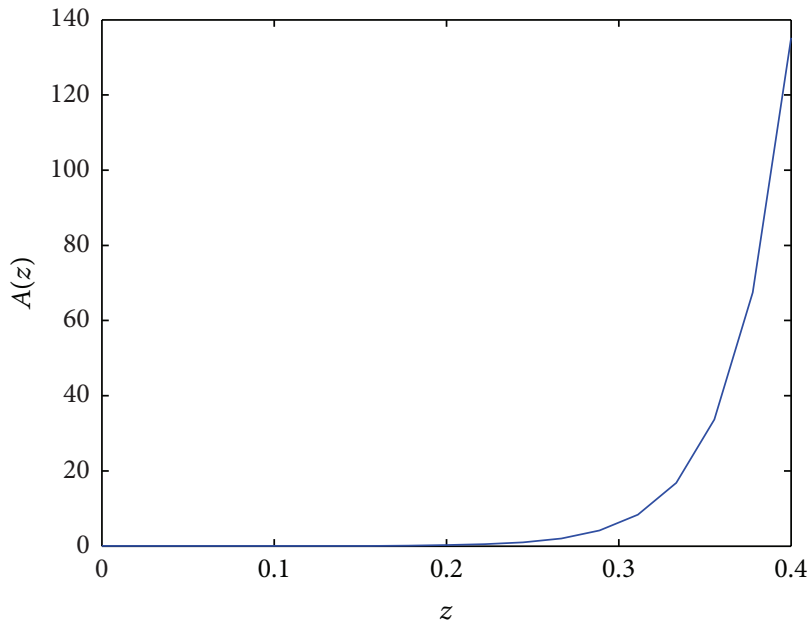

(a)

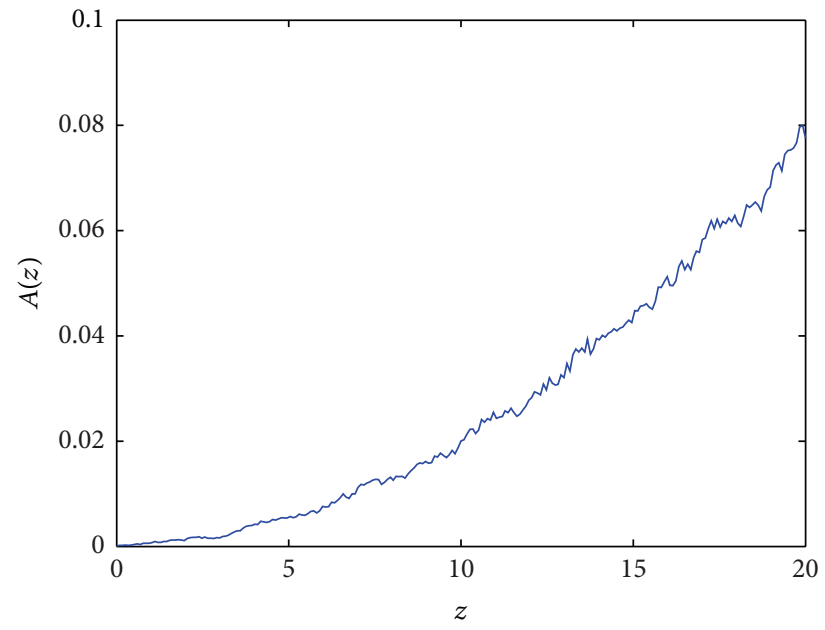

(b)

FIGURE 16: (a) Linear evolution of the lattice soliton with $\mu=1.5$ and $V_{0}=0$. (b) Linear evolution of the lattice soliton on the Penrose lattice with $\mu=1.5$ and $V_{0}=3.5$.

(b) The spectral condition is associated with the eigenvalue problem (see [29]). If the spectral condition is violated it leads to a drift instability; that is, the lattice soliton moves from the potential maximum towards a nearby lattice minimum.

The center of mass of a perturbed soliton is given by

$$
\mathrm{CM}=\frac{1}{P} \int_{-\infty}^{\infty} \int_{-\infty}^{\infty}(x+i y)|u|^{2} d x d y
$$

where the center of mass in $x$ and $y$ coordinates is defined as

$$
\langle x\rangle:=\operatorname{real}(\mathrm{CM}), \quad\langle y\rangle:=\operatorname{imag}(\mathrm{CM}) .
$$

In order to examine the nonlinear stability of the lattice solitons with cubic-quintic nonlinearities, we evaluated the maximum amplitude of the lattice solitons versus the propagation distance, the change in the location of centers of mass. We consider a stable lattice soliton as follows:

(a) it should preserve its peak amplitude, that is, as opposed to finite-distance collapse;

(b) it should preserve its position on the lattice.

If all of the two conditions are satisfied then lattice soliton will be considered nonlinearly stable.

The nonlinear stability of the lattice solitons for the cubicquintic and the quintic model is investigated in the following sections separately.

6.1. Nonlinear Evolution of the Lattice Solitons in the CubicQuintic Model. Until now, we have investigated the linear stability properties of the lattice solitons for the cubic-quintic and the quintic models. None of those solitons for cubicquintic and quintic models are found to be linearly stable. 


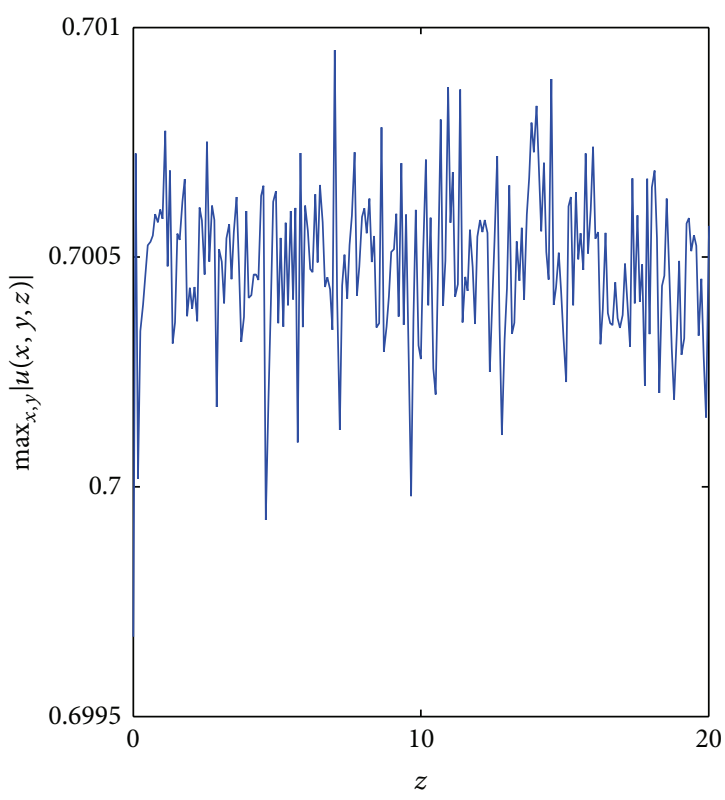

(a)

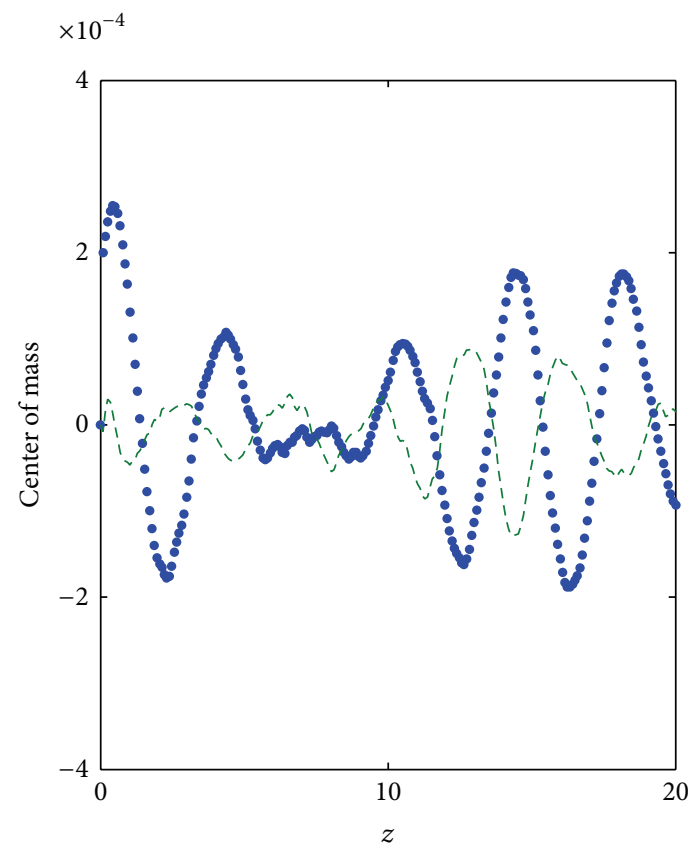

(b)

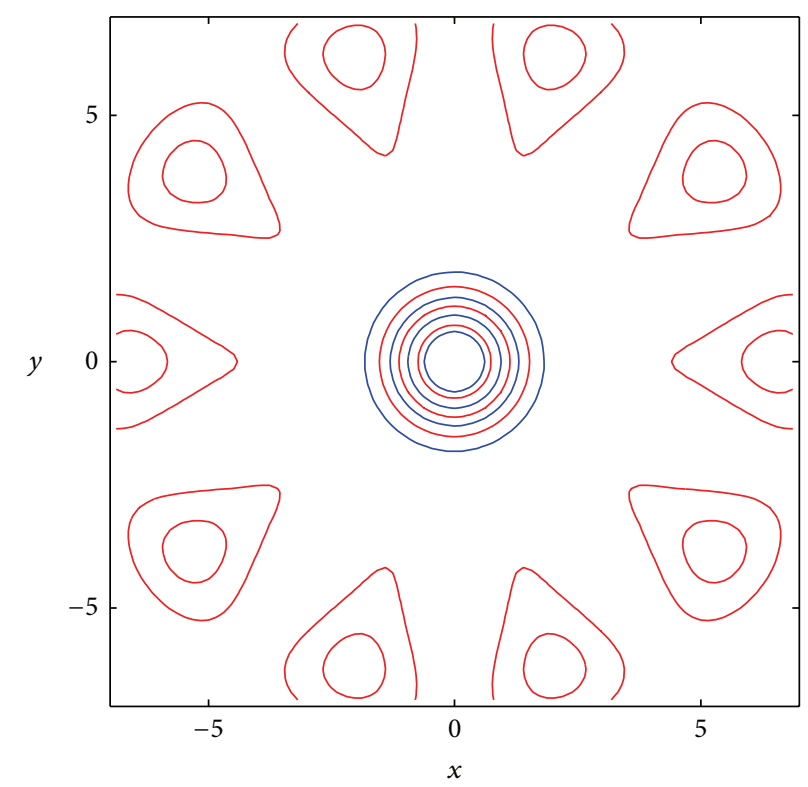

(c)

FIGURE 17: Nonlinear evolution of the lattice soliton corresponding to the first renormalization factor situated at the Penrose potential with $\mu=1.5$ for the self-focusing cubic and the self-defocusing quintic model. (a) Peak amplitude of the lattice soliton as a function of the propagation distance. The initial condition is taken as the lattice soliton corresponding to the first renormalization factor with a 0.01 noise in the amplitude and phase. (b) Center of mass evolution in $x$ and $y$ coordinates. (c) Cross section along the diagonal axis of the fundamental soliton superimposed on the Penrose potential after the propagation $(z=20)$.

We first analyze the nonlinear stability properties of the solitons with the self-focusing cubic and the self-defocusing quintic nonlinearities; that is, $\alpha=1$ and $\beta=-0.2$. In order to examine the nonlinear stability of lattice solitons that were numerically obtained earlier, we plot the maximum amplitude of the lattice solitons obtained by the use of the first and second renormalization factors and center of mass and contour plot of lattice solitons superimposed on the underlying Penrose potential.

As can be seen from Figures 17 and 18 the peak amplitudes of the lattice solitons oscillate with the propagation distance $z$ and the center of mass in the $x$ - and $y$-axis nearly stays at the same place. After the propagation distance $z=20$, the lattice solitons stay at the maximum of the Penrose potential. 


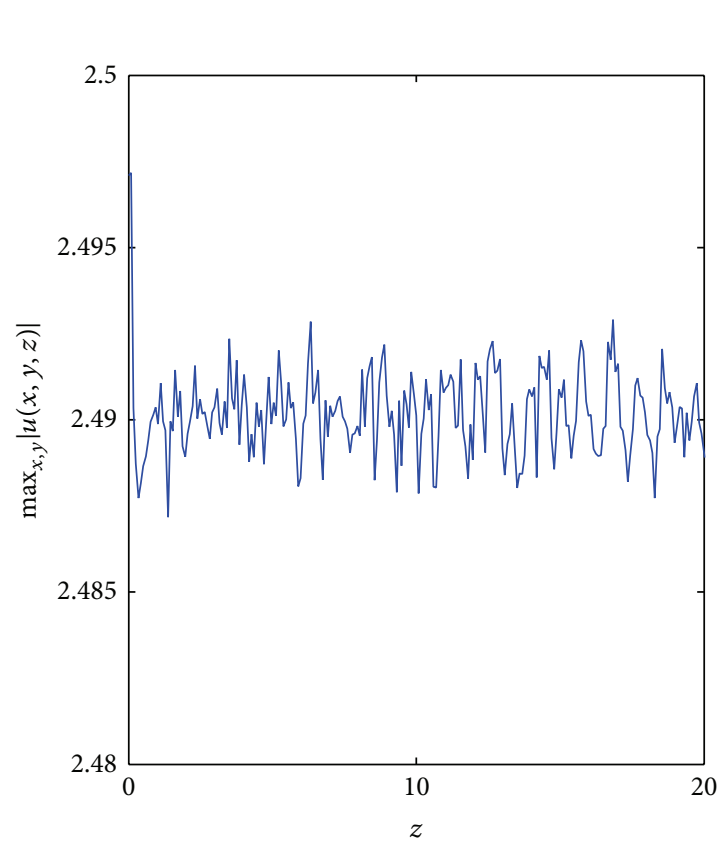

(a)

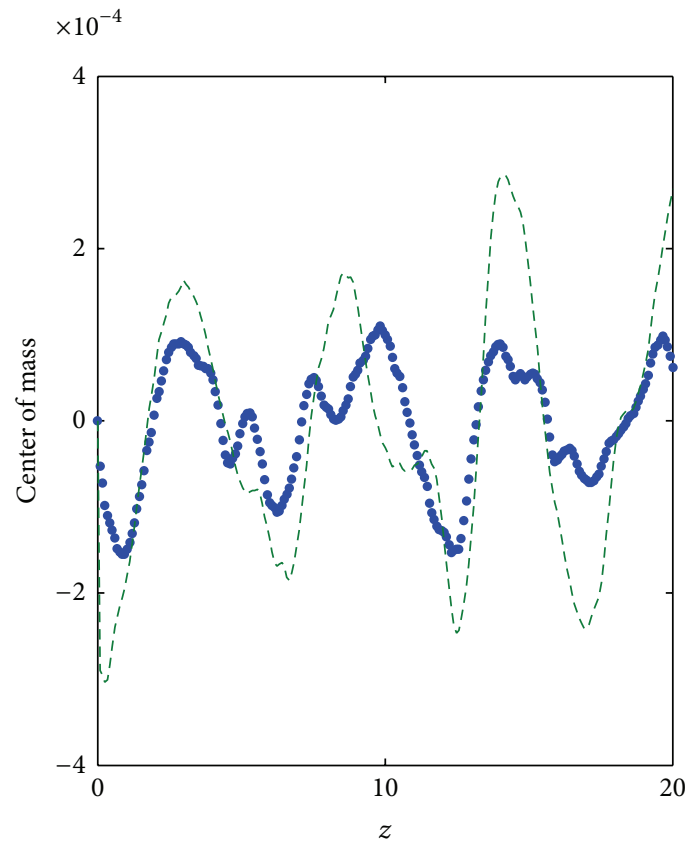

(b)

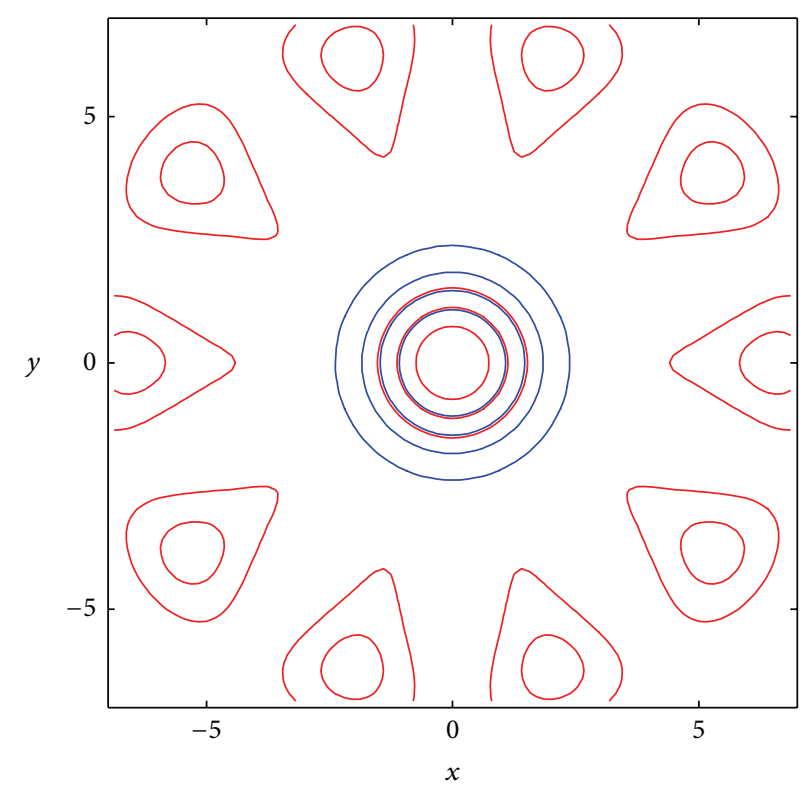

(c)

FIGURE 18: Nonlinear evolution of the lattice soliton corresponding to the second renormalization factor situated at the Penrose potential with $\mu=1.5$ for the self-focusing cubic and the self-defocusing quintic model. (a) Peak amplitude of the lattice soliton as a function of the propagation distance. The initial condition is taken as the lattice soliton corresponding to the first renormalization factor with a 0.01 noise in the amplitude and phase. (b) Center of mass evolution in $x$ and $y$ coordinates. (c) Cross section along the diagonal axis of the fundamental soliton superimposed on the Penrose potential after the propagation $(z=20)$.

This suggests that the lattice solitons obtained by the first and second renormalization factors are nonlinearly stable.

In the second case, we investigate the nonlinear stability properties of the lattice soliton in the case of the self-focusing cubic and self-focusing quintic nonlinearities without the optical lattice. We set $V_{0}=0$ and $\alpha=1, \beta=0.2$.
We see from Figure 19(a), in the case without optical lattice, that soliton is nonlinearly unstable since the maximum amplitude of the soliton increases with the propagation distance. The cross section of the diagonal axis of the lattice soliton stays at the maximum of the Penrose potential after evolution. So there is no drift instability. 


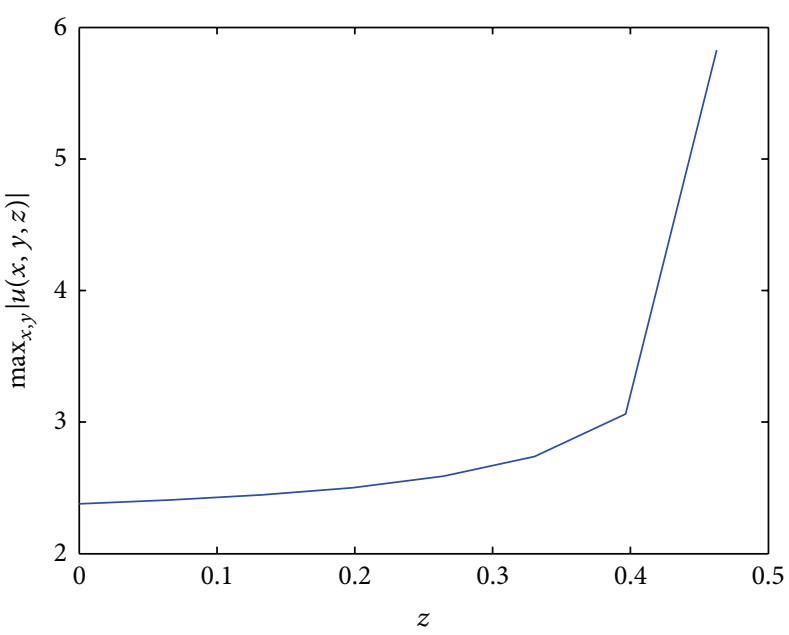

(a)

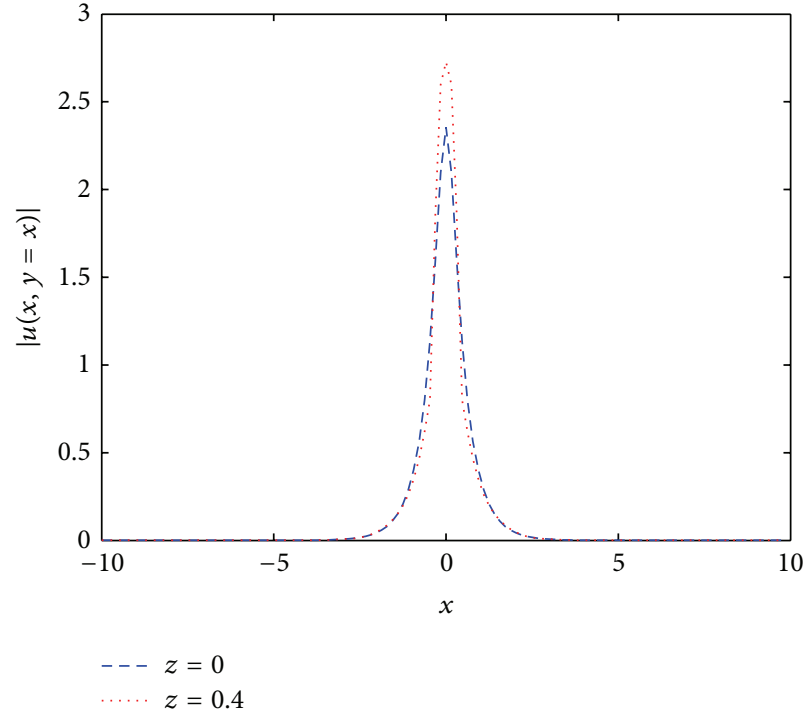

(b)

Figure 19: Nonlinear evolution of the lattice soliton without the optical lattice with $\mu=1.5$ for the self-focusing cubic and the self-focusing quintic model. (a) Peak amplitude of the lattice soliton as a function of the propagation distance. The initial condition is taken as the lattice soliton with a 0.01 noise in the amplitude and phase; (b) cross section along the diagonal axis of the lattice soliton before and after evolutions $(z=0$ and $z=0.4)$.

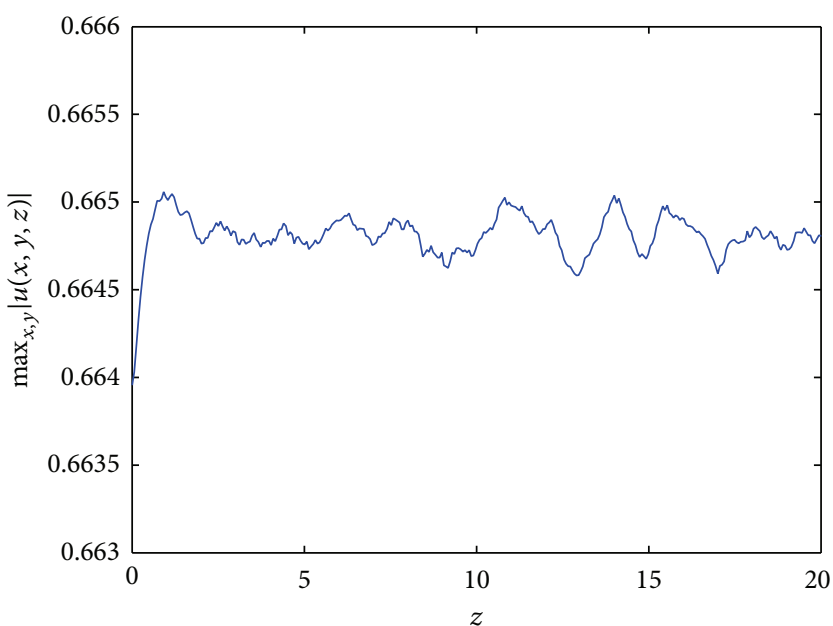

(a)

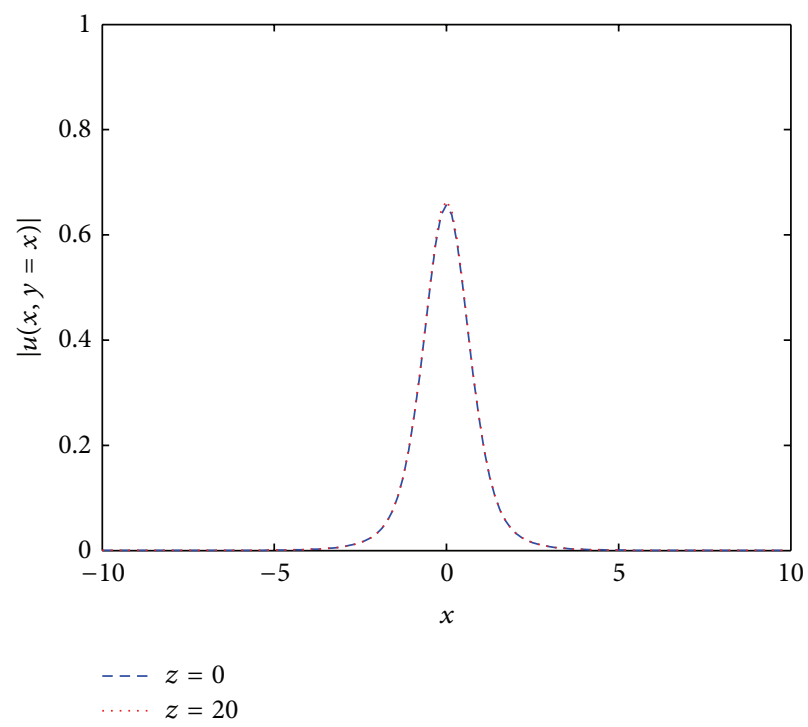

(b)

Figure 20: Nonlinear evolution of the lattice soliton situated at the Penrose potential with $\mu=1.5$ for the self-focusing cubic and the selffocusing quintic model. (a) Peak amplitude of the lattice soliton as a function of the propagation distance. The initial condition is taken as the lattice soliton with a 0.01 noise in the amplitude and phase; (b) cross section along the diagonal axis of the lattice soliton before and after evolutions $(z=0$ and $z=20)$.

The nonlinear stability properties of the lattice soliton centered at the maximum of the Penrose potential for the selffocusing cubic and the self-focusing quintic nonlinearities are displayed in Figure 20. The lattice soliton is nonlinearly stable since the peak amplitude of the lattice soliton slightly oscillates with the propagation distance and the lattice soliton stays at the same place. Numerical investigations show that the collapse occurs when the depth of the potential becomes zero for the self-focusing cubic and the self-focusing quintic model. Thus, adding the optical lattice such as Penrose potential arrests the collapse for the self-focusing cubic and the self-focusing quintic model.

6.2. Nonlinear Evolutions of Lattice Solitons in the Quintic Model. We have investigated the linear stability properties of lattice solitons for the self-focusing quintic nonlinearity in the previous section. The natural question to examine is whether linearly unstable lattice solitons are also nonlinearly 


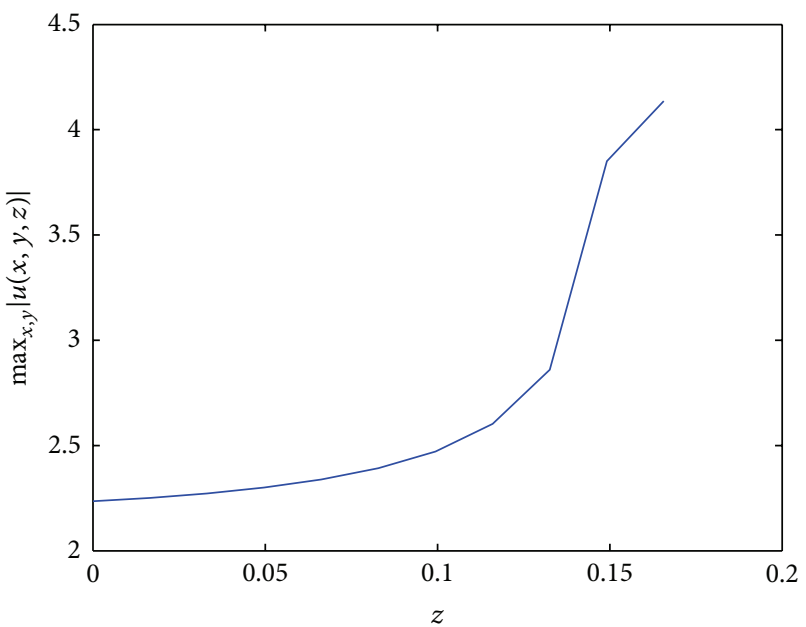

(a)

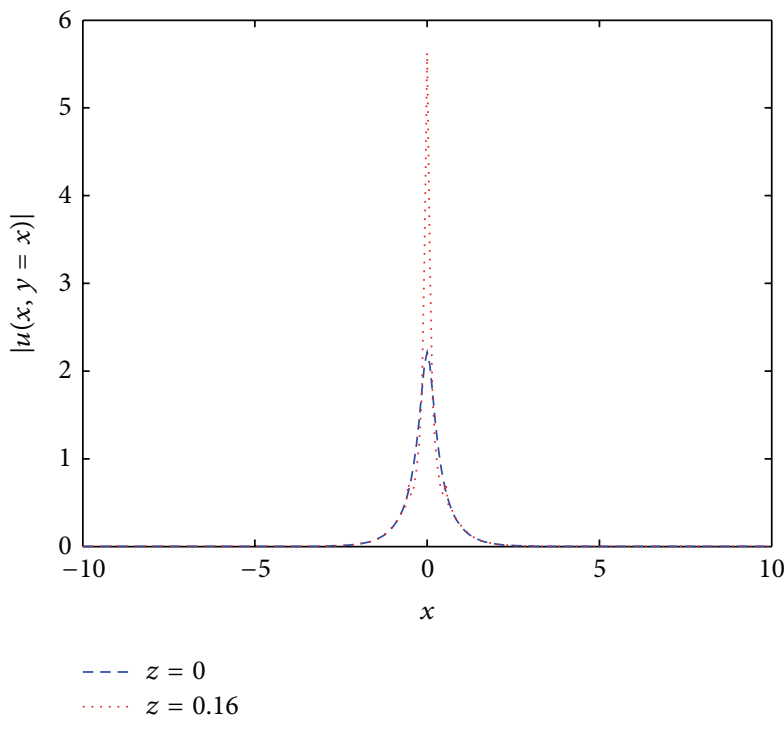

(b)

Figure 21: Nonlinear evolution of the lattice soliton without the optical lattice with $\mu=1.5$ for the self-focusing quintic model. (a) Peak amplitude of the lattice soliton as a function of the propagation distance. The initial condition is taken as the lattice soliton with a 0.01 noise in the amplitude and phase; (b) cross section along the diagonal axis of the lattice soliton before and after evolutions $(z=0$ and $z=0.16)$.

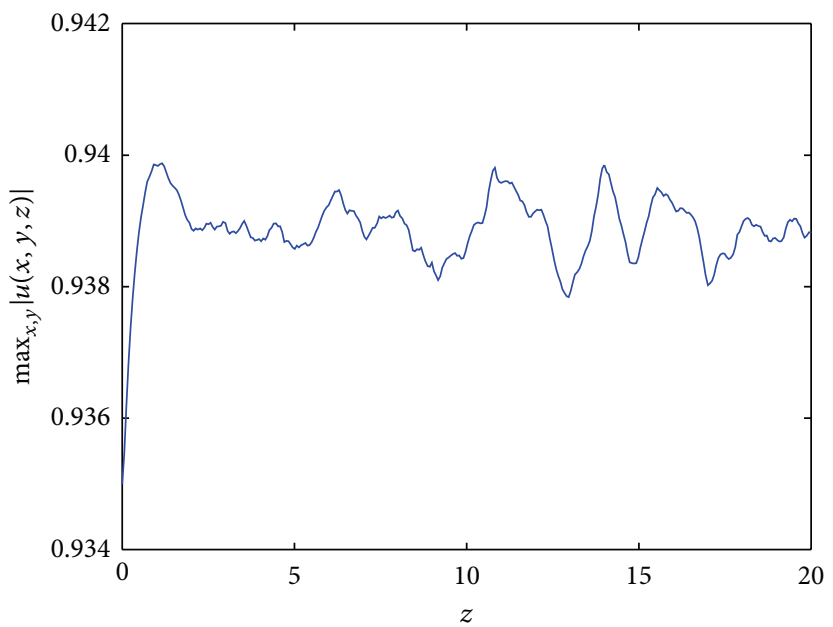

(a)

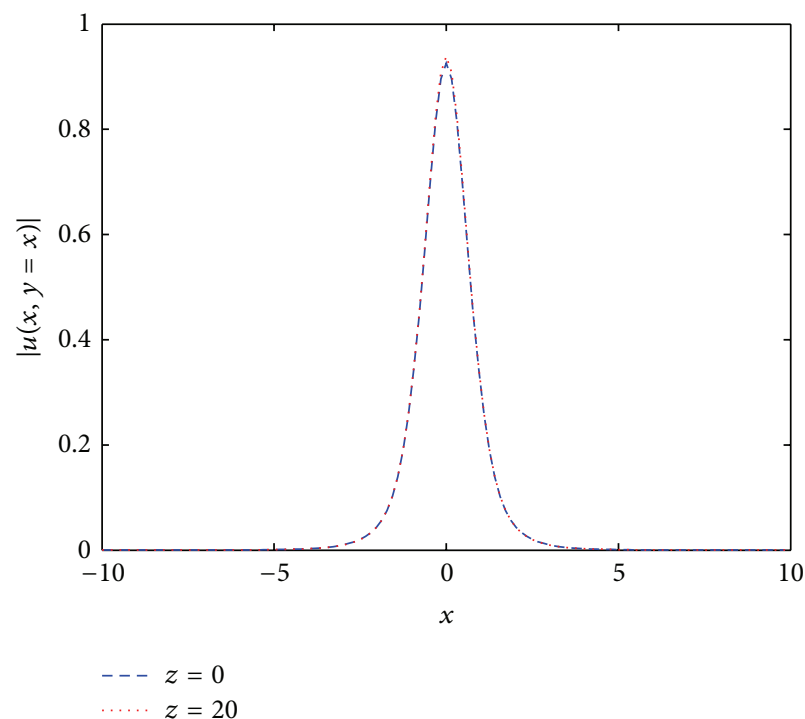

(b)

Figure 22: Nonlinear evolution of the lattice soliton situated at the Penrose potential with $\mu=1.5$ for the self-focusing quintic model. (a) Peak amplitude of the lattice soliton as a function of the propagation distance. The initial condition is taken as the lattice soliton with a 0.01 noise in the amplitude and phase; (b) cross section along the diagonal axis of the lattice soliton before and after evolutions $(z=0$ and $z=20)$.

unstable. In order to examine the nonlinear stability of lattice solitons that we obtained above, we directly compute (2), over a long distance. The initial conditions were taken to be a lattice soliton with 0.01 perturbation in amplitude and phase.

In order to see the effect of the optical lattice on the nonlinear stability, we set $V_{0}=0$ and we take the propagation constant as $\mu=1.5$. That is, there is no external potential. We plotted the maximum amplitude of the lattice soliton versus the propagation distance.

The simulation result is shown in Figure 21. We can see that the lattice soliton quickly blows up; thus it is obviously nonlinearly unstable.
The stability change point exists in the quintic model with the Penrose potential (see Figure 13). The solitons with $d P / d \mu>0$ are stable but solitons with $d P / d \mu<0$ are unstable. In order see whether the solitons with $d P / d \mu>0$ are stable or not we plotted the maximum amplitude of the lattice soliton situated at the Penrose potential versus the propagation distance $z$ and cross section along the diagonal axis of the lattice soliton for small propagation constant, $\mu=1.5$.

It can be seen from Figure 22 that the maximum amplitude of the lattice soliton oscillates with the relatively small amplitude and the lattice soliton stays nearly at the same place during the direct simulation. So there is no drift instability. 


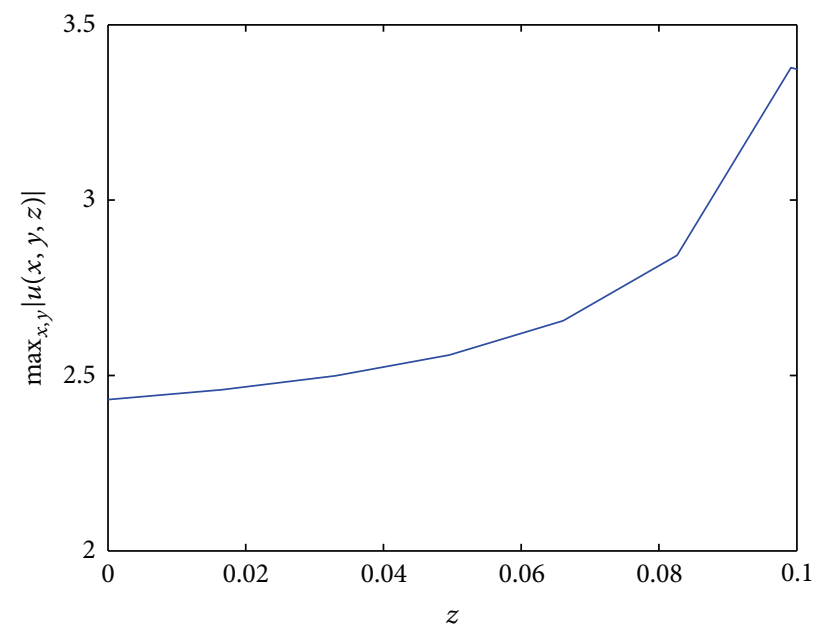

(a)

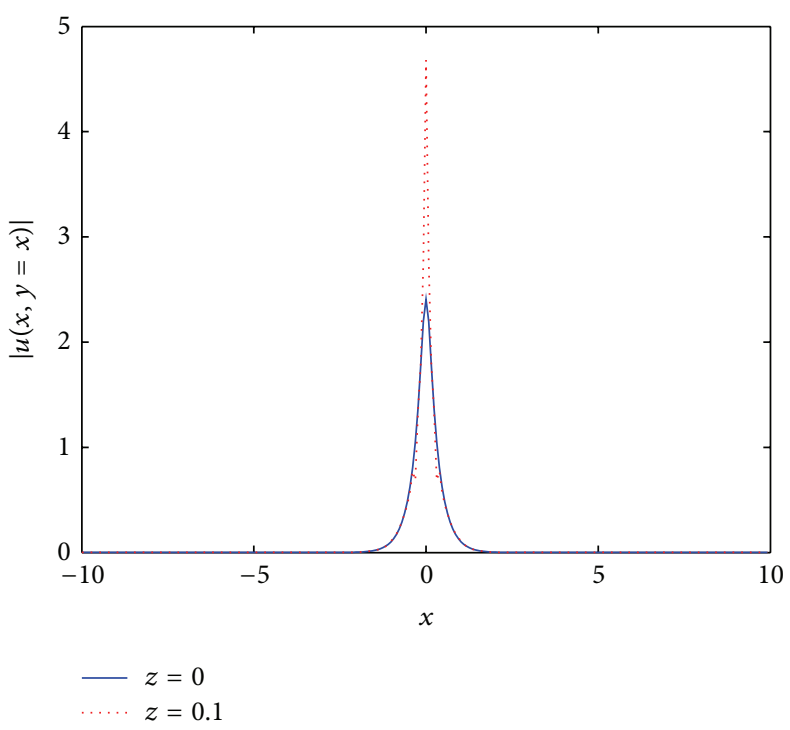

(b)

Figure 23: Nonlinear evolution of the lattice soliton situated at the Penrose potential with $\mu=5$ for the self-focusing quintic model. (a) Peak amplitude of the lattice soliton as a function of the propagation distance. The initial condition is taken as the lattice soliton with a 0.01 noise in the amplitude and phase; (b) cross section along the diagonal axis of the lattice soliton before and after evolutions $(z=0$ and $z=0.1)$.

As a result, by adding the Penrose potential in case of the self-focusing quintic model, the soliton becomes nonlinearly stable. We also plotted the maximum of the lattice soliton with $d P / d \mu<0$ versus the propagation distance. Numerical simulation shows that the lattice soliton with $d P / d \mu<0$ is unstable since the maximum of the lattice soliton increases with the propagation distance. After the evolution, the lattice soliton stays at the maximum of the Penrose potential (see Figure 23).

\section{Conclusion}

In conclusion, we have modified the spectral renormalization method as pseudospectral renormalization method in order to apply different nonlinearities such as saturable nonlinearity. We have numerically demonstrate the existence of two different types of solitons corresponding to the first and second renormalization factors for the self-focusing cubic and the self-defocusing quintic nonlinearities. The self-focusing cubic and the self-defocusing nonlinearities support two different solitons with and without the optical lattice but the selffocusing quintic nonlinearity without the optical lattice does not support two different solitons.

\section{Conflict of Interests}

The author declares that there is no conflict of interests regarding to the publication of this paper.

\section{References}

[1] Y. Kivshar and G. P. Agrawal, Optical Solitons: From Fibers to Photonic Crstals, Academic Press, San Diego, Calif, USA, 2003.

[2] W. van Saarloos and P. C. Hohenberg, "Pulses and fronts in the complex Ginzburg-Landau equation near a subcritical bifurcation," Physical Review Letters, vol. 64, no. 7, pp. 749-752, 1990.
[3] B. A. Malomed and A. A. Nepomnyashchy, "Onset of chaos in the generalized Ginzburg-Landau equation," Physical Review A, vol. 42, no. 10, pp. 6238-6240, 1990.

[4] L.-C. Crasovan, B. A. Malomed, and D. Mihalache, "Stable vortex solitons in the two-dimensional Ginzburg-Landau equation," Physical Review E, vol. 63, Article ID 016605, 2001.

[5] R. Scharf and A. R. Bishop, "Length-scale competition for the one-dimensional nonlinear Schrödinger equation with spatially periodic potentials," Physical Review E, vol. 47, no. 2, pp. 1375$1383,1993$.

[6] J. C. Bronski, L. D. Carr, B. Deconinck, and J. N. Kutz, "BoseEinstein condensates in standing waves: the cubic nonlinear Schrödinger equation with a periodic potential," Physical Review Letters, vol. 86, no. 8, pp. 1402-1405, 2001.

[7] J. C. Bronski, L. D. Carr, B. Deconinck, J. N. Kutz, and K. Promislow, "Stability of repulsive Bose-Einstein condensates in a periodic potential," Physical Review E, vol. 63, no. 3, Article ID 036612, 2001.

[8] J. C. Bronski, L. D. Carr, R. Carretero-González, B. Deconinck, J. N. Kutz, and K. Promislow, "Stability of attractive Bose-Einstein condensates in a periodic potential," Physical Review E, vol. 64, no. 5, Article ID 056615, 9 pages, 2001.

[9] C. Sulem and P. Sulem, The Nonlinear Schrödinger Equation: Self-Focusing and Wave Collapse, Springer, Berlin, Germany, 2000 .

[10] D. N. Christodoulides, F. Lederer, and Y. Silberberg, "Discretizing light behaviour in linear and nonlinear waveguide lattices," Nature, vol. 424, no. 6950, pp. 817-823, 2003.

[11] A. A. Sukhorukov, Y. S. Kivshar, H. S. Eisenberg, and Y. Silberberg, "Spatial optical solitons in waveguide arrays," IEEE Journal of Quantum Electronics, vol. 39, no. 1, pp. 31-50, 2003.

[12] N. K. Efremidis, J. Hudock, D. N. Christodoulides, J. W. Fleischer, O. Cohen, and M. Segev, "Two-dimensional optical lattice solitons," Physical Review Letters, vol. 91, no. 21, Article ID 213906, 2003. 
[13] J. W. Fleischer, M. Segev, N. K. Efremidis, and D. N. Christodoulides, "Observation of two-dimensional discrete solitons in optically induced nonlinear photonic lattices," Nature, vol. 422, no. 6928 , pp. 147-150, 2003.

[14] M. J. Ablowitz, N. Antar, I. BakIrtaş, and B. Ilan, "Band-gap boundaries and fundamental solitons in complex two-dimensional nonlinear lattices," Physical Review A, vol. 81, no. 3, Article ID 033834, 2010.

[15] C. T. Zhou and X. T. He, "Stochastic diffusion of electrons in evolutive Langmuir fields," Physica Scripta, vol. 50, no. 4, pp. 415-418, 1994.

[16] T. A. Davydova, A. I. Yakimenko, and Y. A. Zaliznyak, “Twodimensional solitons and vortices in normal and anomalous dispersive media," Physical Review E, vol. 67, no. 2, Article ID 026402, 16 pages, 2003.

[17] I. V. Barashenkov and V. G. Makhankov, "Soliton-like "bubbles" in a system of interacting bosons," Physics Letters A, vol. 128, no. 1-2, pp. 52-56, 1988.

[18] A. Bulgac, "Dilute quantum droplets," Physical Review Letters, vol. 89 , no. $5,2002$.

[19] F. K. Abdullaev, A. Gammal, L. Tomio, and T. Frederico, "Stability of trapped Bose-einstein condensates," Physical Review A, vol. 63, no. 4, Article ID 043604, 14 pages, 2001.

[20] N. Akhmediev and A. Ankiewics, Dissipative Solitons, Springer, Berlin, Germany, 2005.

[21] M. G. Clerc, S. Coulibaly, M. A. Garcia-Ñustes, and Y. Zárate, "Dissipative localized states with shieldlike phase structure," Physical Review Letters, vol. 107, no. 25, Article ID 254102, 2011.

[22] M. J. Ablowitz, B. Ilan, E. Schonbrun, and R. Piestun, "Solitons in two-dimensional lattices possessing defects, dislocations, and quasicrystal structures," Physical Review E: Statistical, Nonlinear, and Soft Matter Physics, vol. 74, no. 3, 2006.

[23] B. Freedman, G. Bartal, M. Segev, R. Lifshitz, D. N. Christodoulides, and J. W. Fleischer, "Wave and defect dynamics in nonlinear photonic quasicrystals," Nature, vol. 440, no. 7088, pp. 1166-1169, 2006.

[24] M. J. Ablowitz and Z. H. Musslimani, "Spectral renormalization method for computing self-localized solutions to nonlinear systems," Optics Letters, vol. 30, no. 16, pp. 2140-2142, 2005.

[25] M. J. Ablowitz, T. P. Horikis, and B. Ilan, "Solitons in dispersionmanaged mode-locked lasers," Physical Review A, vol. 77, no. 3, Article ID 033814, 2008.

[26] M. J. Ablowitz, N. Antar, A. BakIrtaş, and B. Ilan, "Vortex and dipole solitons in complex two-dimensional nonlinear lattices," Physical Review A: Atomic, Molecular, and Optical Physics, vol. 86, no. 3, Article ID 033804, 2012.

[27] N. G. Vakhitov and A. A. Kolokolov, "Stationary solutions of the wave equation in a medium with nonlinearity saturation," Radiophysics and Quantum Electronics, vol. 16, no. 7, pp. 783789, 1975.

[28] Y. B. Gaididei, J. Schjødt-Eriksen, and P. L. Christiansen, "Collapse arresting in an inhomogeneous quintic nonlinear Schrödinger model," Physical Review E, vol. 60, no. 4 B, pp. 48774890, 1999.

[29] B. Ilan, Y. Sivan, and G. Fibich, "A quantitative approach to soliton instability," Optics Letters, vol. 36, no. 3, pp. 397-399, 2011. 


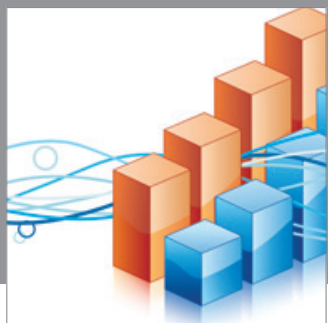

Advances in

Operations Research

mansans

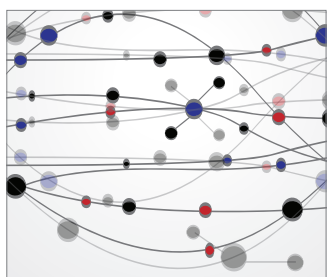

The Scientific World Journal
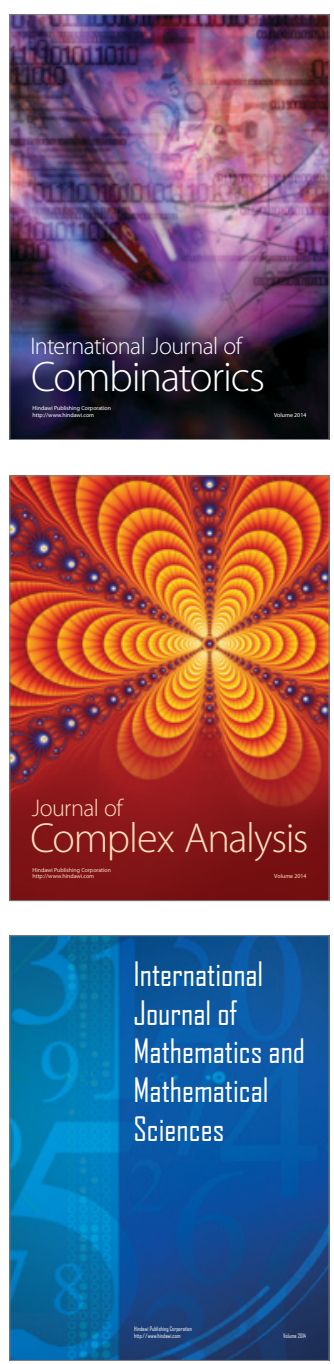
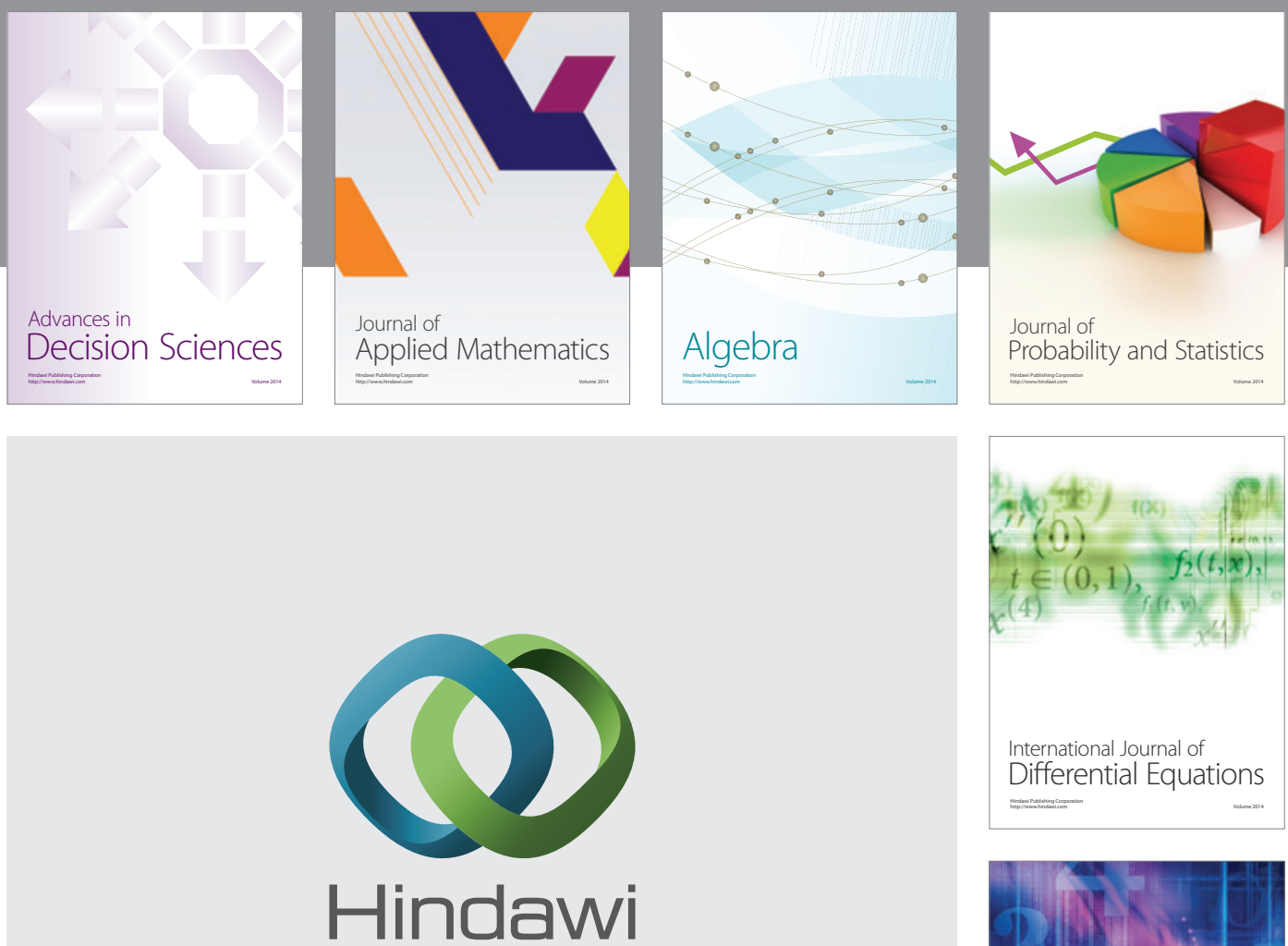

Submit your manuscripts at http://www.hindawi.com
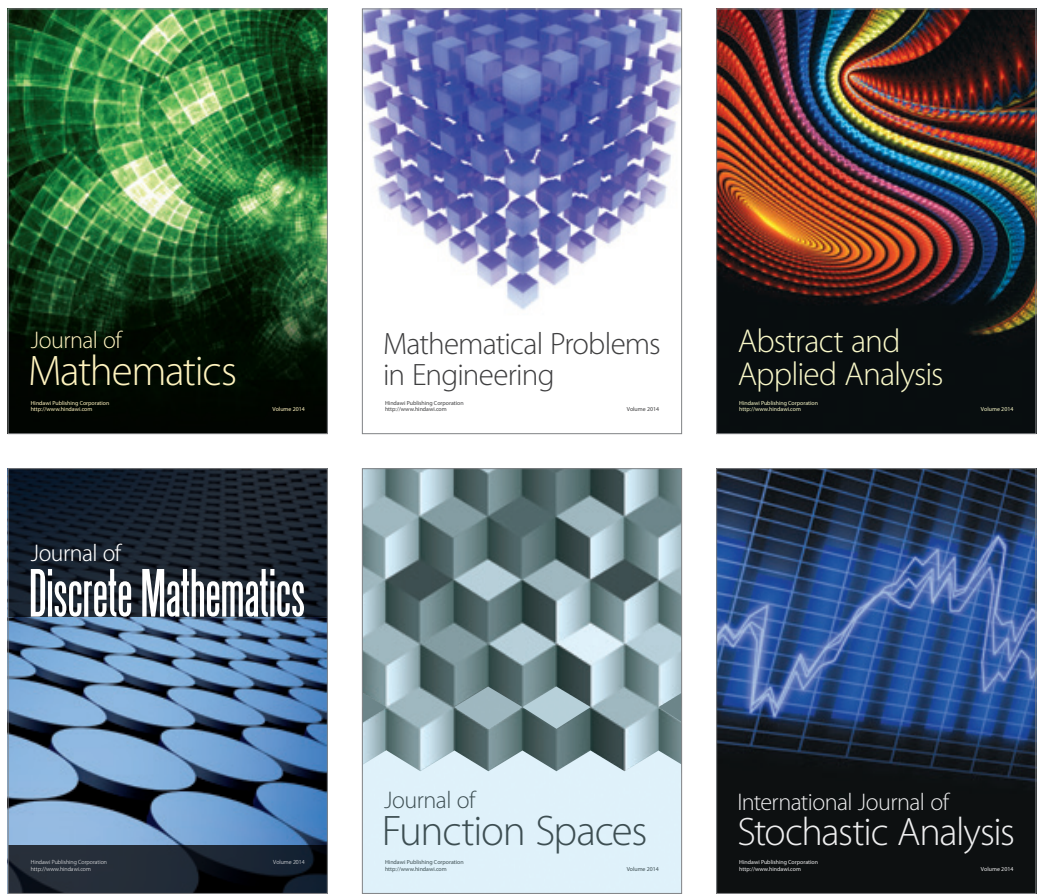

Journal of

Function Spaces

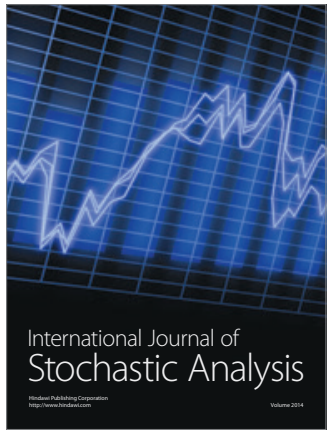

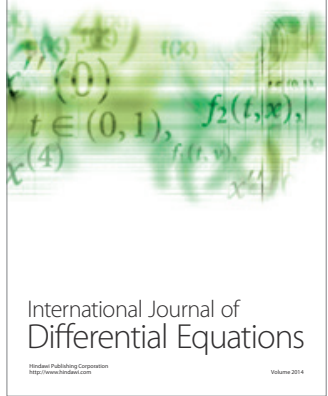
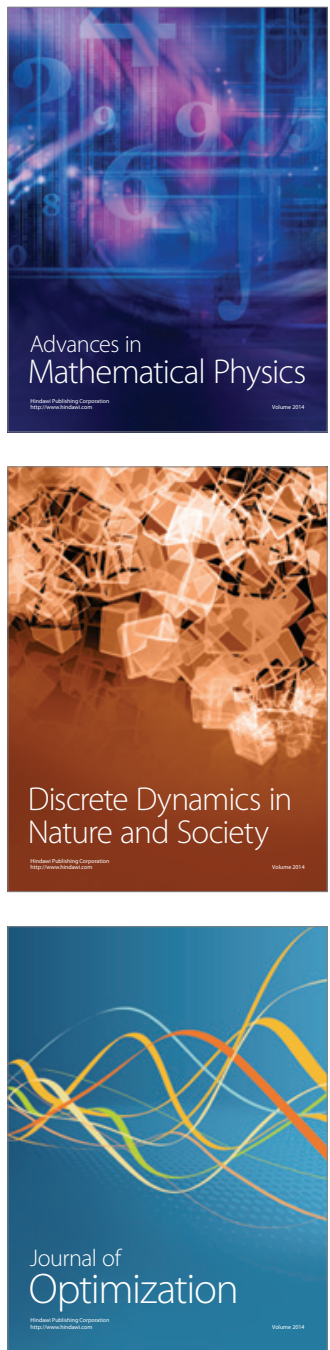